\title{
MUSE sneaks a peek at extreme ram-pressure events ${ }^{\star}$
}

\section{Tomography of UGC 6697, a massive galaxy falling into Abell 1367}

\author{
G. Consolandi ${ }^{1}$, G. Gavazzi ${ }^{1}$, M. Fossati ${ }^{2,3}$, M. Fumagalli ${ }^{4}$, A. Boselli ${ }^{5}$, M. Yagi ${ }^{6,7}$, and M. Yoshida ${ }^{8}$ \\ 1 Università degli Studi di Milano-Bicocca, Piazza della Scienza 3, 20126 Milano, Italy \\ e-mail: guido. consolandi@mib.infn.it \\ 2 Max-Planck-Institut für Extraterrestrische Physik, Giessenbachstrasse, 85748 Garching, Germany \\ 3 Universitäts-Sternwarte München, Schenierstrasse 1, 81679 München, Germany \\ 4 Institute for Computational Cosmology, and Centre for Extragalactic Astronomy, Durham University, South Road, Durham, \\ DH1 3LE, UK \\ 5 Aix-Marseille Univ., CNRS, LAM, Laboratoire d'Astrophysique de Marseille, 130313 Marseille, France \\ 6 Optical and Infrared Astronomy Division, National Astronomical Observatory of Japan, Mitaka, 181-8588 Tokyo, Japan \\ 7 Graduate School of Science and Engineering, Hosei University, 3-7-2, Kajinocho, Koganei, 184-8584 Tokyo, Japan \\ 8 Hiroshima Astrophysical Science Center, Hiroshima University, 1-3-1, Kagamiyama, Higashi-Hiroshima, 739-8526 Hiroshima, \\ Japan
}

Received 22 May 2017 / Accepted 19 July 2017

\begin{abstract}
We present the MUSE observations of UGC 6697, a giant $\left(M_{*} \approx 10^{10} M_{\odot}\right)$ spiral galaxy infalling in the nearby cluster Abell 1367 During its high-velocity transit through the intracluster medium (ICM), the hydrodynamical interactions with the ICM produce a $\approx 100 \mathrm{kpc}$ tail of ionized gas that we map with a mosaic of five MUSE pointings up to $60 \mathrm{kpc}$ from the galaxy. CGCG 97087N, a small companion that lies at few arcminutes in projection from UGC 6697, is also showing signs of the hydrodynamic action of the ICM of the cluster. Along the whole extent of the tail, we detect diffuse $\mathrm{H} \alpha$ emission, and to a lesser extent, $\mathrm{H} \beta$, [OIII] $\lambda 5007$, and [OI] 26300 . By comparing the kinematics and distribution of gas and stars (as traced by the CaII triplet) for both galaxies, we separate the ionized gas, as traced by the $\mathrm{H} \alpha$ line, into a component that is still bound to the galaxy and a component that is stripped. We find that the bound component shows a low-velocity dispersion and line ratios consistent with photoionization by hot stars. The stripped gas is more turbulent, with velocity dispersions up to $\gtrsim 100 \mathrm{~km} \mathrm{~s}^{-1}$, and is excited by shocks, as traced by high values of [OI]/H $\alpha$ and $[\mathrm{NII}] / \mathrm{H} \alpha$ ratio. In the tail of UGC 6697, we identify numerous bright compact knots with line ratios typical of HII regions. These are distributed along the only streams of stripped gas that retain low-velocity dispersions $\left(\lesssim 35 \mathrm{~km} \mathrm{~s}^{-1}\right)$. Despite being in the stripped gas, their physical properties are not different from normal HII regions in galactic disks. We find evidence of a past fast encounter between the two galaxies in the form of a double tail emerging from CGCG 97087N that connects with UGC 6697. This encounter might have increased the efficiency of the stripping process, leaving the stellar distribution and kinematics unaltered.
\end{abstract}

Key words. galaxies: evolution - galaxies: clusters: individual: UGC 6697 - galaxies: interactions - galaxies: star formation

\section{Introduction}

The evolutionary path that drives galaxies through the cosmic ages from being actively star forming to "red and dead" systems is regulated by the complex interplay between two broad classes of processes that are able to remove (or rapidly consume) the reservoir of cold gas, thus quenching the galaxies star formation: i) internal (or secular) processes that are driven by the mass (dubbed "downsizing"; Fontanot et al. 2009; Gavazzi 2009; Siudek et al. 2017); and ii) external processes that are driven by the environment in which galaxies are embedded. As galaxies approach regions of high density such as groups or clusters, environmental processes start to sum up the secular

\footnotetext{
* The composite data cube is only available at the CDS via anonymous ftp to cdsarc.u-strasbg. fr (130.79.128.5) or via http://cdsarc.u-strasbg.fr/viz-bin/qcat?J/A+A/606/A83
}

ones and strongly contribute to the build-up of the red and passive population of galaxies (Dressler 1980; Boselli \& Gavazzi 2006).

Environmental processes can be further divided into two classes: gravitational interactions (tidal interaction - harassment; Moore et al. 1996) and hydrodynamic interactions between the hot intracluster medium (ICM) and the interstellar medium (ISM) of galaxies infalling at high velocity in clusters (ram pressure, Gunn \& Gott 1972; viscous stripping, Nulsen 1982; thermal evaporation, Cowie \& McKee 1977; and starvation, Larson 1980, see Boselli \& Gavazzi 2006, 2014, for reviews). However, the relative importance of the different processes shaping galaxies in dense environments is still poorly defined. The direct observations of environmental interactions has often been hampered by technological limitations. All multiwavelength studies aiming at directly detecting environmental processes demand a high sensitivity as well as sufficient resolution. Apart 
from striking merging events, shells and stellar streams triggered by mergers are tenuous features (see Duc et al. 2015) characterized by low surface brightness emission. Similarly, the diffuse gas stripped by hydrodynamic interactions is characterized by emission of very low surface brightness (Yoshida et al. 2004; Sun et al. 2007; Chung et al. 2009; Boselli et al. 2016; Fumagalli et al. 2014; Fossati et al. 2016; Merluzzi et al. 2016).

The local Universe is obviously the best place to reach high sensitivities with high resolution in a reasonable integration time. On the other hand, local galaxies have apparent sizes of several arcminutes, thus requiring large fields of view (Yoshida et al. 2004; Boselli et al. 2016). Hence, for many years, the direct observations of the environmental interactions has been limited to a few targeted objects, preventing a statistical analysis. Recently, however, we entered a new era. The advent of new imaging cameras and integral field unit (IFUs) spectrographs is a turning point for the study of environmental effects. In visible light, telescopes such as CFHT and Subaru now mount cameras with large fields of view (FOVs, $\approx 1 \mathrm{deg}^{2}$ ) and are equipped with large interferometric filters, which enables mapping large areas.

For instance, using SuprimeCam mounted on the Subaru telescope, Yagi et al. (2010) and Yagi et al. (2017) have mapped the ionized gas emission as traced by $\mathrm{H} \alpha$ of galaxies in the Coma and Abell 1367 clusters with unprecedented spatial resolution and sensitivity. They reported that stripping phenomena are ubiquitous in these clusters because a large fraction of latetype galaxies (LTGs) are observed in the process of loosing their ionized gas through their hydrodynamic interaction with the ICM. More recently, Boselli et al. (2016), using the MegaCam mounted on the CFHT telescope, targeted with deep H $\alpha$ observations a massive spiral in the Virgo cluster (NGC 4569) and found a spectacular $75 \mathrm{kpc}$ tail trailing behind the galaxy. These observations are particularly interesting because they vividly show that ram-pressure stripping may be very effective even in massive galaxies inside a young and intermediate-mass cluster, such as Virgo (Binggeli et al. 1987).

In this new era, IFUs represent a breakthrough in the study of environment. As shown by the recent studies of Fumagalli et al. (2014), Fossati et al. (2016), Merluzzi et al. (2016), Poggianti et al. (2017), Bellhouse et al. (2017), and Fritz et al. (2017), IFUs are able to recover with high spatial resolution the dynamics of both gas and stars and to provide all the spectroscopic information required to constrain the physical state of the ISM and of the stellar component. Many IFU followup observations of galaxies interacting in high-density regions are scheduled (Poggianti et al. 2017), allowing us to build sufficient statistics to explore characteristic trends in the physical state of systems interacting with the environment.

The present work analyzes the MUSE observations of the spiral galaxy UGC 6697, which is infalling in the nearby cluster Abell 1367. Unlike the galaxy ESO 137-001 (the very first studied with this instrument, Fumagalli et al. 2014; Fossati et al. 2016), UGC 6697 is a massive galaxy $\left(M_{*} \approx 10^{10} M_{\odot}\right)$ transiting edge-on through the ICM of the cluster. This configuration should, in principle, disfavor the ram-pressure stripping. A small companion, CGCG $97087 \mathrm{~N}$, lies at just few arcseconds in projection away from UGC 6697, and a recent Subaru observation revealed double gaseous tails connecting to UGC 6697, which indicates a possible galaxy-galaxy interaction. This system, differently from ESO 137-001, offers the opportunity of studying a triple interaction (ICM-galaxy-satellite).

The layout of the paper is as follows. In Sect. 2 we present the targets (UGC 6697 and CGCG 97087N) by summarizing results from the literature. In Sects. 3 and 4 we provide the details of our observations, of the data reduction, and of the software we used to analyze the final data product. The results are presented in Sect. 5, with a full kinematical analysis in Sects. 5.1 and 5.2. The analysis of the physical state of the ionized gas using line ratios is presented in Sect. 6.1. We further constrain the ionization state of the gas by building spatially resolved BPT diagrams (Baldwin et al. 1981) in Sect. 6.2, and we extract the properties of the HII regions observed in the tails in Sect. 7. Finally, in Sect. 8 we discuss our results.

\section{Targets}

UGC 6697 (also known as CGCG 97087, Nilson 1973; Zwicky et al. 1968) is the second brightest spiral/Irr member $\left(c z=6727 \mathrm{~km} \mathrm{~s}^{-1}\right)$ of the nearby cluster Abell $1367(\langle c z\rangle=$ $\left.6595 \mathrm{~km} \mathrm{~s}^{-1}\right)$. It is located at RA $=11^{\mathrm{h}} 43^{\mathrm{m}} 49 \mathrm{~s} .03$ and Dec $=$ $+19^{\circ} 58^{\prime} 05^{\prime \prime} .5$, at a projected distance of $462 \mathrm{kpc}$ to the NW from the X-ray center of Abell $1367\left(\mathrm{RA}=11^{\mathrm{h}} 44^{\mathrm{m}} 36.5\right.$ and Dec $=$ $+19^{\circ} 45^{\prime} 32^{\prime \prime}$, according to Piffaretti et al. 2011), well within the virial radius of the cluster (2.13 Mpc; Girardi et al. 1998). The region of the cluster that is inhabited by UGC 6697 is shown in the Subaru field of Fig. 1. A smaller companion (CGCG 97087N) with a recessional velocity of $c z=7542 \mathrm{~km} \mathrm{~s}^{-1}$ lies at $\sim 20 \operatorname{arcsec}$ to the NE from UGC 6697, while NGC $3842\left(c z=6247 \mathrm{~km} \mathrm{~s}^{-1}\right)$, one of the two giant E galaxies of Abell 1367, lies at 3.5 arcmin to the E. The small offset between the velocity of UGC 6697 and of the cluster suggests that the galaxy is crossing Abell 1367 nearly in the plane of the sky (at the assumed distance of 94.8 Mpc of Abell 1367, 1 arcsec separation corresponds to $0.46 \mathrm{kpc}$, and one arcmin to $27.8 \mathrm{kpc}$ ). In contrast, the velocity of CGCG $97087 \mathrm{~N}$ suggests that its motion is almost along the line of sight.

The galaxies UGC 6697 and CGCG $97087 \mathrm{~N}$ have a stellar mass of $\approx 10^{10} M_{\odot}$ and $\approx 10^{9} M_{\odot}$ respectively, as computed from the $i$-band luminosity and the $(g-i)$ color following Zibetti et al. (2009).

We were alerted to UGC 6697 by the head-tail morphology of the radio continuum source associated with it (Gavazzi 1978). This morphology, which is generally found in elliptical cluster galaxies, has not been found previously in a spiral galaxy. This feature suggests that the galaxy is under the action of ram pressure (Gunn \& Gott 1972) during its high-velocity edge-on transit through the cluster ICM. Many multifrequency observations targeting UGC 6697 have been gathered after its discovery. From 1984 to 1989 , the galaxy has been observed in $\mathrm{H} \alpha$ (Bothun \& Schommer 1984), radio, and optical continuum (Gavazzi et al. 1984), at $1415 \mathrm{MHz}$ (Gavazzi \& Jaffe 1987), and in HI (Gavazzi 1989). All observations consistently show signs of the cometary shape of UGC 6697 and mutually reinforce the interpretation of this object based on the ram-pressure scenario. More recently, Sun \& Vikhlinin (2005), using the Chandra observatory, detected significant X-ray diffuse emission that shows a morphological asymmetry similar to the emission observed in the radio, hence suggesting an ongoing hydrodynamic interaction with the ICM of Abell 1367. Based on the early work of Nulsen (1982), these authors note that the geometry of the impact of the galaxy with the ICM (edge-on) should disfavor ram pressure as the main hydrodynamic process at play. Instead, viscous or turbulent stripping should be the principle hydrodynamic process that is stripping the gas from UGC 6697.

However, variations of the ram pressure scenario were also proposed, mainly because of the multiple velocity components along the line of sight in the rotation curve of UGC 6697 


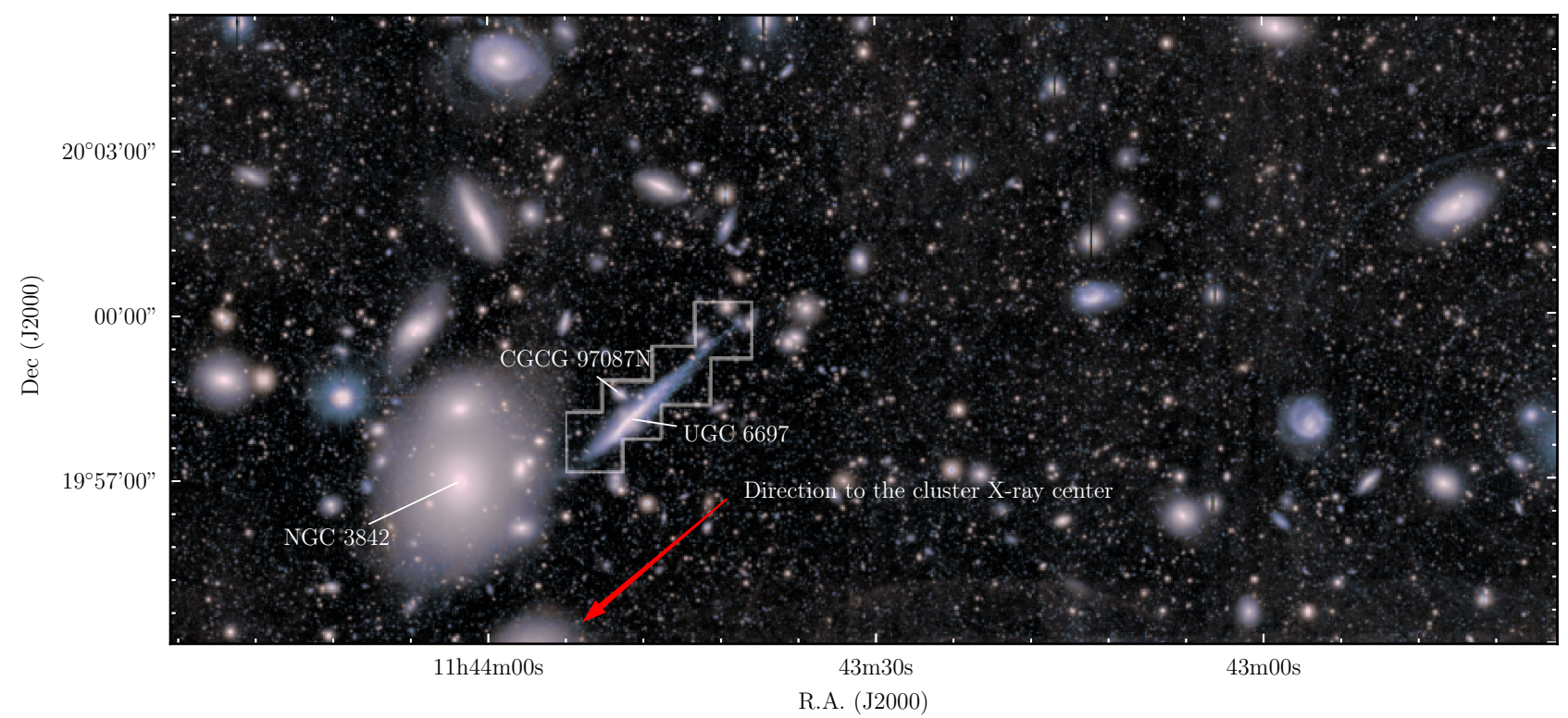

Fig. 1. RGB (iRB) image of the central region of Abell 1367 containing NGC 3842, one of the two giant E galaxies in this cluster (left), the highly inclined UGC 6697 and its faint companion CGCG $97087 \mathrm{~N}$, which are the subject of the present investigation. The gray line indicates the positions of the MUSE mosaic. The observations were taken with the Subaru observatory by Yagi et al. (2017).

Table 1. Details of the observing blocks composing the mosaic of UGC 6697 ordered in right ascension from E to W.

\begin{tabular}{ccccccc}
\hline \hline OB & $\begin{array}{c}\text { RA } \\
(\mathrm{hh} \mathrm{m} \mathrm{s})\end{array}$ & $\begin{array}{c}\text { Dec } \\
\left({ }^{\prime}{ }^{\prime \prime}\right)\end{array}$ & $\begin{array}{c}\text { Obs date } \\
(\mathrm{dd} \mathrm{mm} \mathrm{yy})\end{array}$ & $\begin{array}{c}T \exp \\
(\mathrm{s})\end{array}$ & AM & $\begin{array}{c}\text { Seeing } \\
(\operatorname{arcsec})\end{array}$ \\
\hline 11 & 114351.46 & 195742.5 & 290216 & $2 \times 2190$ & $1.48-1.57$ & $0.77-1.07$ \\
1 & 114348.70 & 195816.8 & 100216 & $2 \times 2190$ & $1.40-1.45$ & $1.01-1.36$ \\
7 & 114348.70 & 195816.8 & 100216 & $2 \times 2190$ & $1.51-1.62$ & $0.72-1.95$ \\
2 & 114345.00 & 195853.0 & 100216 & $2 \times 2190$ & $1.40-1.44$ & $1.00-1.90$ \\
8 & 114341.85 & 195940.0 & 290216 & $2 \times 2190$ & $1.40-1.43$ & $1.02-1.43$ \\
\hline
\end{tabular}

(Gavazzi et al. 1984, 2001). Gavazzi et al. (1984) speculated that a possible collision between UGC 6697 and CGCG $97087 \mathrm{~N}$ might have occurred, causing a flow of gas to emerge from UGC 6697, while Gavazzi et al. (2001) speculated that the complex morphology and kinematics of the galaxy was the result of the merging of two different systems. Recently, Yagi et al. (2017), using the $8 \mathrm{~m}$ Subaru telescope, obtained a deep $\mathrm{H} \alpha$ image of Abell 1367, including UGC 6697. They discovered that its low surface brightness $\mathrm{H} \alpha$ cometary trail extends toward the NW for up to $100 \mathrm{kpc}$. Surprisingly, they also found clear evidence of a faint gaseous double-tail that emerges perpendicularly from the disk of CGCG 97087N and connects to the disk of UGC 6697, supporting the scenario proposed by Gavazzi et al. (1984).

\section{Observations}

The galaxy UGC 6697 and its companion CGCG 97087N were observed with MUSE on the VLT (UT4) on February 10 and 29, 2016, as part of proposal 096.B-0019(A) (PI Gavazzi). A mosaic of the five MUSE pointings taken at the coordinates listed in Table 1 is shown in Fig. 2. These pointings cover from the optical front of the galaxy (SE of the mosaic) up to $54 \mathrm{kpc}$ outside the optical extent of the galaxy in the NW, where the $\mathrm{H} \alpha$ tail extends. Each observation consisted of two 21.5 min exposures obtained by rotating the instrument by 90 degrees, interspersed by an empty-sky exposure of 4 min duration, taken at the coordinates RA $=11^{\mathrm{h}} 43^{\mathrm{m}} 41^{\mathrm{s}} ;$ Dec $=+19^{\circ} 54^{\prime} 52^{\prime \prime}$. The central pointing that covers the center of UGC 6697 and its companion
CGCG $97087 \mathrm{~N}$ was repeated (OB1 and OB7) in order to reach a higher signal-to-noise ratio $(\mathrm{S} / \mathrm{N})$ in the area of a possible interaction between the two. Observations were conducted while the galaxy was transiting at airmass $\sim 1.5$ in clear sky conditions, and seeing ranging from 0.7 to 1.9 arcsec, using the Wide Field Mode with nominal wavelength coverage (4800-9300 ̊). Flux calibration was achieved by means of observing the standard star GD153, exposed for $160 \mathrm{~s}$.

\section{Data reduction and analysis}

The final datacube mosaic was obtained using the MUSE data reduction pipeline (v 1.2.1) and a set of Python-based codes that we designed in order to improve the quality of the illumination correction and of the sky subtraction for the observed field.

For each exposure composing the mosaic as well as for each sky and standard star frames, the first step was to create a master bias, master dark, a master flat, and a wavelength solution within the MUSE pipeline. These were applied to each science and sky cube and to the standard star (GD153). After computing and applying the telluric correction spectrum within the MUSE pipeline, we reconstructed the final cubes for each exposure. In order to produce a mosaic containing five different pointings, we constructed an astrometry reference frame by building a regular 2D-WCS grid that maps all the exposures of the final mosaic. Starting from the WCS information contained in the reference WCS frame, we built for each exposure a regular 3D-WCS grid with a $0.2^{\prime \prime}$ spatial pixel scale and a $1.25 \AA$ spectral step 


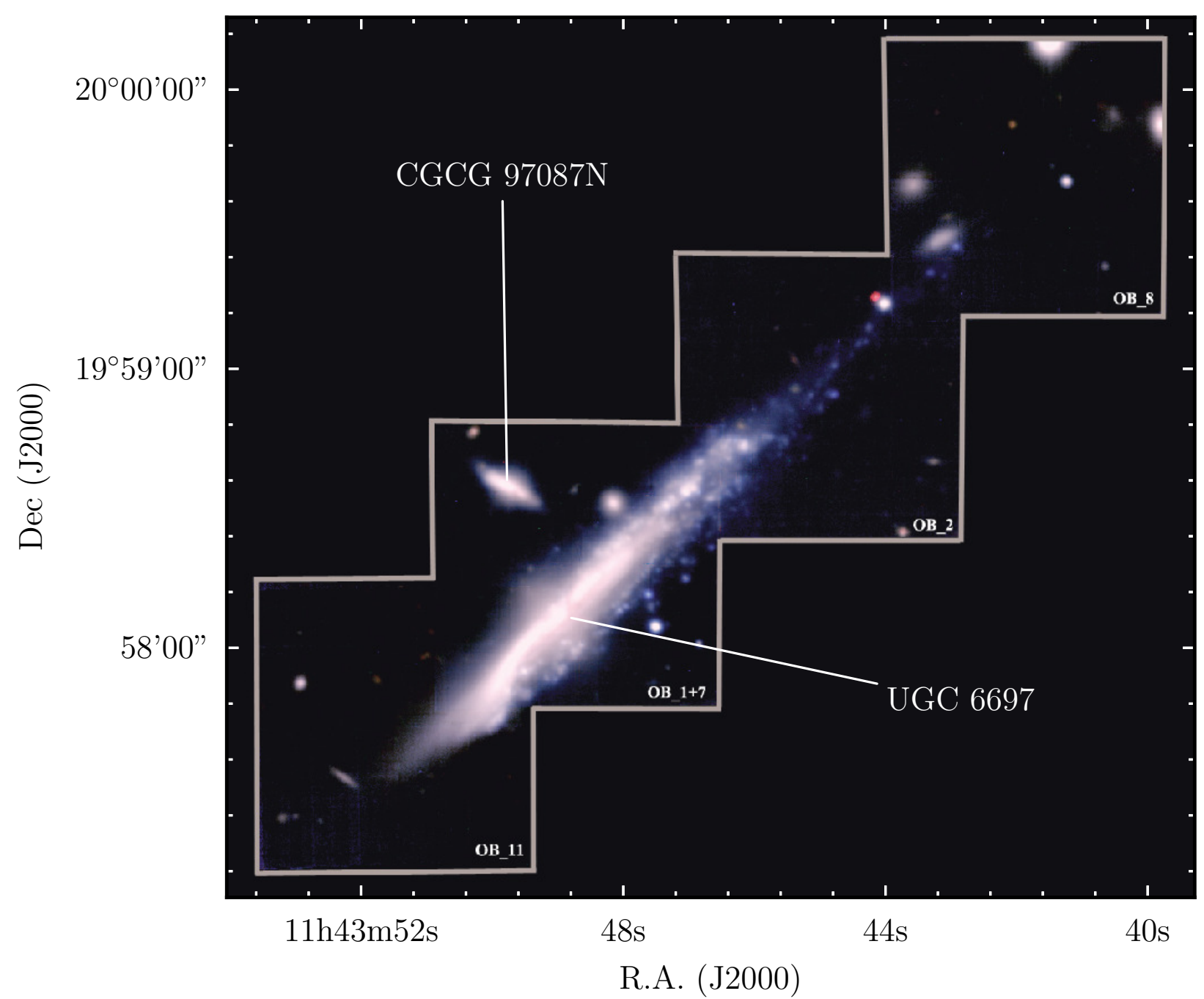

Fig. 2. False color image of the 5 mosaicked MUSE pointings that cover UGC 6697. Its companion galaxy UGC 6697N lies at $\sim 20$ arcsec projected distance in the NE direction. Each RGB channel was constructed from the reduced MUSE data cube by projecting the cube respectively in the $g$, $r$ and $i$ SDSS bands.

from 4750 to $9300 \AA$ A. All science exposures are projected onto their 3D grid using the MUSE pipeline task muse_scipost. Every reconstructed cube of each exposure was mapped onto the same wavelength and spatial grid.

The reconstructed cubes exhibit a residual variation in the illumination of each IFU that depends on the wavelength at which the FOV is observed. In order to perform a robust sky subtraction that minimizes the residuals (Fumagalli et al. 2014, 2017), we applied to each sky and science exposure an illumination correction. This was computed using in-house Python codes that evaluate, after masking bright contaminating objects, the illumination correction as a function of wavelength by computing the response of each IFU compared to the average response of the field in intervals of $500 \AA$.

After correcting the illumination of each sky and science fields, we obtained a master sky spectrum by collapsing onto the wavelength axis the single sky exposure that is free of contaminating sources and properly masked. To construct a correct model of the sky spectrum that accounts for both the time dependency of air glow line and continuum fluxes, we used SKYCORR (Noll et al. 2014) in the same way as in Fossati et al. (2016). The sky model was then subtracted from each spaxel by interpolating the spectrum on the wavelength axis with a spline function. The uncertainties were appropriately added to the variance extension of the datacube.

After sky subtraction, a single datacube for each pointing was created by combining the respective exposures, and the final datacube was assembled following the mosaic map. The final result is shown in the RGB of Fig. 2, which was constructed by convolving the datacube with the SDSS gri filters. The noise background, in the central field covered by four expositions, has a $1 \sigma$ limiting surface brightness around the redshifted $\mathrm{H} \alpha$ line $(\lambda \sim 6680-6750 \AA)$ of $\approx 5 \times$ $10^{-19} \mathrm{erg} \mathrm{s}^{-1} \mathrm{~cm}^{-2} \operatorname{arcsec}^{-2} \AA^{-1}$. In the other fields, the sky surface brightness reaches $\approx 9.5 \times 10^{-19} \mathrm{erg} \mathrm{s}^{-1} \mathrm{~cm}^{-2} \operatorname{arcsec}^{-2} \AA^{-1}$. Around the redshifted $\mathrm{H} \beta$ line $(\lambda \sim 4930-5015 \AA)$, these values become $\approx 1 \times 10^{-18} \mathrm{erg} \mathrm{s}^{-1} \mathrm{~cm}^{-2} \operatorname{arcsec}^{-2} \AA^{-1}$ and $\approx 2 \times$ $10^{-18} \mathrm{erg} \mathrm{s}^{-1} \mathrm{~cm}^{-2} \operatorname{arcsec}^{-2} \AA^{-1}$, respectively. In order to test the flux calibration reached within our datacubes, we compared the flux of few point-like sources in the field with their corresponding flux in the SDSS. We found a consistency within $\approx 3 \%$.

In order to characterize the properties of the ionized gaseous component of the galaxies, we extracted flux maps of the emission lines listed in Table 2, while in order to trace the kinematics of the stellar component, we fit the second line of the Ca II triplet at 8542.09 $\AA$. To robustly estimate emission line 
Table 2. Emission lines considered in this study.

\begin{tabular}{ccc}
\hline \hline Line & $\begin{array}{c}\lambda \\
(\AA)\end{array}$ & $\begin{array}{c}\mu_{\min } \\
\left(\mathrm{erg} \mathrm{s}^{-1} \mathrm{~cm}^{-2} \AA^{-1} \mathrm{arcsec}^{-2}\right)\end{array}$ \\
\hline $\mathrm{H} \beta$ & 4861.33 & $\approx 5.3-9.7 \times 10^{-18}$ \\
{$[\mathrm{OIII}]$} & 4658.81 & $\approx 4.9-9.1 \times 10^{-18}$ \\
{$[\mathrm{OIII}]$} & 5006.84 & $\approx 4.9-9.1 \times 10^{-18}$ \\
{$[\mathrm{OI}]$} & 6300.30 & $\approx 4.1-5.6 \times 10^{-18}$ \\
{$[\mathrm{NII}]$} & 6548.05 & $\approx 3-4.6 \times 10^{-18}$ \\
$\mathrm{H} \alpha$ & 6562.82 & $\approx 3-4.6 \times 10^{-18}$ \\
{$[\mathrm{NII}]$} & 6583.45 & $\approx 3-4.6 \times 10^{-18}$
\end{tabular}

Notes. Column (1) lists the name of the emission lines and Col. (2) the respective wavelengths in air. Column (3) represent the surface brightness limits at $5 \sigma$ from the central cube covered by four exposures and the surface brightness limits at $5 \sigma$ for the other cubes.

fluxes, we accounted for the stellar absorptions underlying the Balmer emission lines that fall in the MUSE spectral window: $\mathrm{H} \alpha$ and $\mathrm{H} \beta$. For this, we used the code GANDALF (Sarzi et al. 2006) complemented by the penalize pixel-fitting code (Cappellari \& Emsellem 2004) to simultaneously model the stellar continuum and the emission lines in individual spaxels with $S / N>5$. The stellar continuum was modeled with the superposition of stellar templates from the MILES library (Vazdekis et al. 2010) convolved by the stellar line-of-sight velocity distribution, whereas the emission lines and kinematics were modeled assuming a Gaussian profile. In each spaxel, the modeled stellar continuum spectrum was subtracted from the observed spectrum to obtain a final datacube of pure emission lines that is free of stellar absorption.

In order to account for more complex kinematics along the line of sight (previously described by Gavazzi et al. 2001), we designed a Python fitting code called $Q B f i t$. QBfit allows fitting groups of emission lines (or line sets) at once, such as $\mathrm{H} \alpha$ and [NII] $\lambda \lambda 6548,6584 \AA$, by fitting 1D Gaussian functions with the relative velocity separation of the lines kept fixed according to the wavelengths listed in Table 2. The fit of [OIII] and [NII] line sets assumes the ratio between the [OIII] lines and between the [NII] lines the ratio is fixed to the values published in Storey \& Zeippen (2000). The continuum is evaluated locally with a flat power-law, and the $\mathrm{S} / \mathrm{N}$ ratio of the $\mathrm{H} \alpha$ is evaluated using the "stat" extension of the datacube. Moreover, QBfit allows the fit of an additional line set with a different velocity component by simultaneously fitting the same line set twice with a shift in wavelength. In the end, the code returns kinematics and flux maps separated for a low-velocity and a high-velocity component.

We fit the emission lines in the continuum free datacube, assuming a systemic redshift of $z_{\text {sys }}=0.02243$ for UGC 6697 and after a median smoothing of $10 \times 10$ spaxel $(2 \operatorname{arcsec})$ in the spatial dimension to increase the $\mathrm{S} / \mathrm{N}$ ratio per pixel. We did not perform any smoothing along the spectral dimension. During the fitting procedure, we masked sky line residuals and spaxels where the $\mathrm{S} / \mathrm{N}$ of the $\mathrm{H} \alpha$ line was lower than 5. Additional masking was applied to spaxels in which the line centroid or the line width were extremely deviant or showed an error greater than $50 \mathrm{~km} \mathrm{~s}^{-1}$.

The double velocity component fit was not necessary across the whole galaxy extension, but the procedure automatically recognized the spaxels that show double velocities by fitting a single velocity component and by using the width of the emission
Vel $\mathrm{H} \alpha=60.8(\mathrm{~km} / \mathrm{s})$
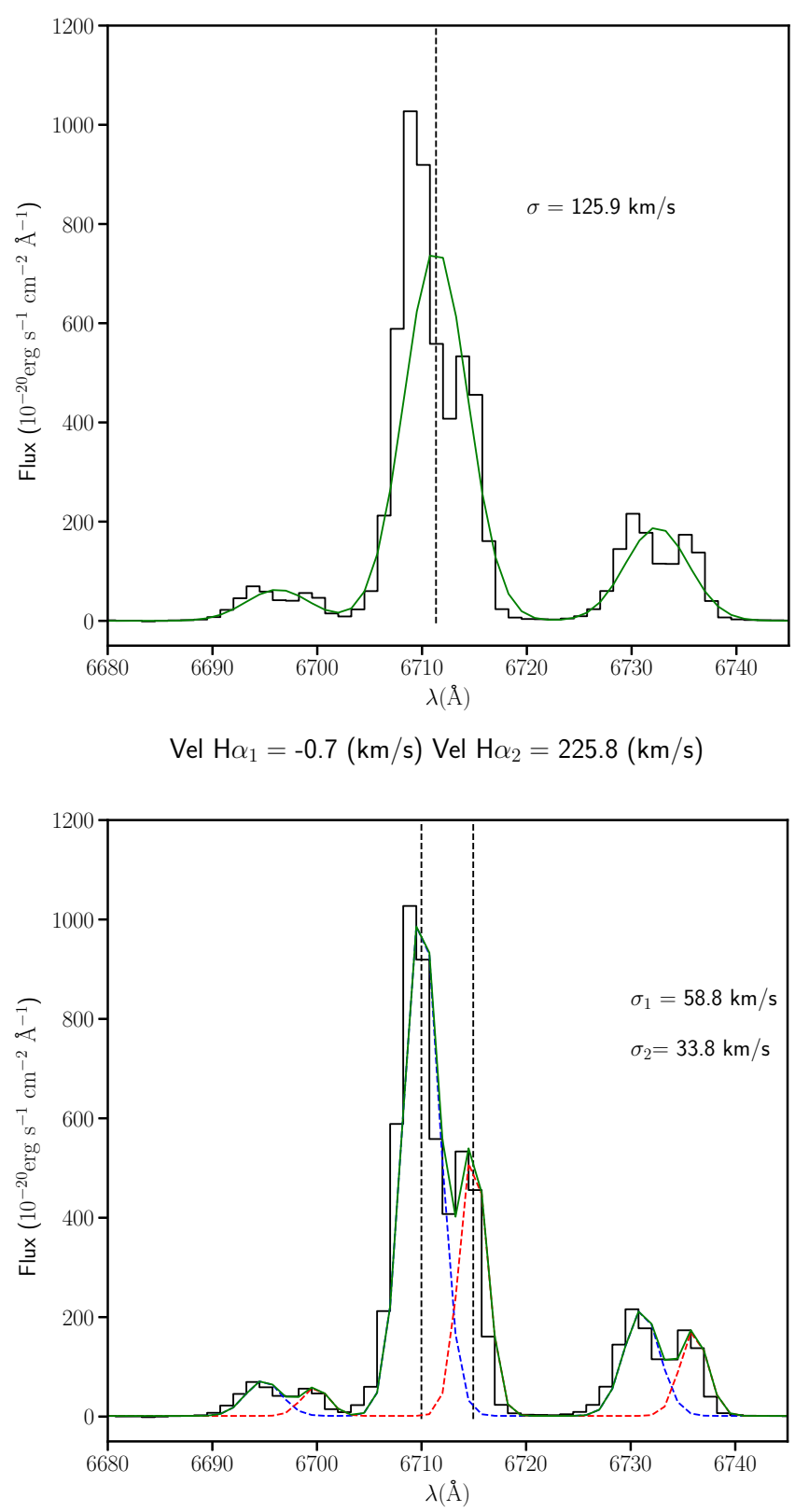

Fig. 3. Spectrum around $\mathrm{H} \alpha$ of a spaxel clearly showing two velocity components. In the top panel, the single-velocity fit to the data is shown in green, while in the bottom panel the sum of the two-velocity component fit is shown in green. The individual velocity components are displayed with blue and red lines.

line as a proxy for multiple velocity components. The width was evaluated after taking into account the instrumental width of the line, evaluated given the MUSE spectral resolution function. As an example, Fig. 3 shows the spectrum around the $\mathrm{H} \alpha$ emission line of a spaxel, clearly showing two velocity components (as evident from the double horn profile of the $\mathrm{H} \alpha$ and [NII] lines) fit with a single velocity component (top) and with two velocity components (bottom) by $Q B f i t$. The fit that accounts for just one velocity component finds a solution with a very large velocity dispersion $\left(\approx 125 \mathrm{~km} \mathrm{~s}^{-1}\right)$. A better fit is obtained when two velocity components are considered. We set the sigma threshold to distinguish when a double velocity fit may be needed to $35 \mathrm{~km} \mathrm{~s}^{-1}$, which is the typical velocity dispersion 
of the gaseous component in galaxies at $z \sim 0$ (Wisnioski et al. 2015).

After a double velocity component fit was performed, $Q B f i t$ performed additional checks in order to limit false or ambiguous detections: the two components must be separated by at least $1.25 \AA$, which is the size of a pixel; both components must have $S / N>5$ in order to represent a detection of a velocity component. In each spaxel, when the solution did not satisfy these conditions or when the double velocity component fit did not converge, the result of the single velocity component fit was stored instead. As a rule, $Q B f$ it always stored the single velocity solution as part of the high-velocity component.

To evaluate the stellar kinematics, we performed a Gaussian fit to the second line of the CaII triplet $(\lambda 8542.09 \AA)$ after smoothing the datacube by $10 \times 10$ spaxels.

\section{Flux and kinematics of gas and stars}

In this section we discuss the kinematics of stars and gas as evaluated from the Ca II absorption line ( $18542.09 \AA)$ and the $\mathrm{H} \alpha+[\mathrm{NII}]$ emission lines, respectively, exploring the results of the two velocity component fit performed assuming the systemic redshift of UGC 6697 for the whole frame (hence CGCG $97807 \mathrm{~N}$ is not centered at $0 \mathrm{~km} \mathrm{~s}^{-1}$ ). In order to test the robustness of our double component fits, we compared this to results obtained with KUBEVIZ (see Fossati et al. 2016) and found good agreement. The low- and high-velocity maps, together with stellar kinematics, are shown in Fig. 4. The distribution of the spaxels that contain two velocity components is traced by the low-velocity component, while the high-velocity map contains the spaxel fit with one velocity as well.

The first two panels show that the gas distribution of UGC 6697 extends to the NW for twice the extent of the stellar component traced by the gray contours of the continuum in the $r$ filter. The extent and orientation of the tail suggest that UGC 6697 is traveling at high speed, almost in the plane of the sky, from NW to SE, and it shows effects of the hydrodynamic interaction with the hot intacluster medium (ICM). In the SE front of the stellar component of the galaxy, the gas is absent and no emission line is detected. The tail extends approximately for $50 \mathrm{kpc}$, including many HII regions (visible also in Fig. 2), seen preferentially in the upper edge of the tail. In Fig. 5 we show the X-ray contours of Sun \& Vikhlinin (2005) superimposed on our map of the total $\mathrm{H} \alpha$ flux. The X-ray emission is coplanar to the $\mathrm{H} \alpha$ : at the front, where the ISM impacts the ICM, the contours compress and form a sharp edge that corresponds to the edge observed in $\mathrm{H} \alpha$; in the NW, both tails overcome the length of the optical galaxy. We refer to the radius at which we observe this sharp edge as the observed stripping radius. By considering the ICM density measured by Sun \& Vikhlinin $\left(2005 ; \rho_{\mathrm{ICM}} \approx 4.5 \times 10^{-4} \mathrm{~cm}^{-3}\right)$ at the position of UGC 6697, we can evaluate the ram pressure for different velocities. On the other hand, we evaluate the anchoring force of UGC 6697 as a function of the radius as to be $\Pi=2 \pi G \Sigma_{\text {gas }} \Sigma_{\text {stars }}$, where $\Sigma_{\text {gas }}$ and $\Sigma_{\text {stars }}$ are modeled with a simple exponential profile of the form $\Sigma=\left(M_{\mathrm{d}} /\left(2 \pi r_{\mathrm{d}}^{2}\right)\right) \mathrm{e}^{-r / r_{\mathrm{d}}}$, where $M_{\mathrm{d}}$ is the mass and $r_{\mathrm{d}}$ the disk scale length. We assume $M_{\mathrm{d} \text {,stars }}=10^{10.5} M_{\odot}$ and $M_{\mathrm{d}, \mathrm{gas}}=0.19 \times M_{\mathrm{d} \text {,stars }}$ (as evaluated from the HI mass measured by Gavazzi et al. 2001) and an $r_{\mathrm{d}} \approx 5 \mathrm{kpc}$, typical of a $10^{10.5} M_{\odot}$ disk galaxy at $z \sim 0$ (Courteau et al. 2007). By comparing the ram pressure obtained with different velocities to the anchoring force as a function of the radius, we can reproduce the observed stripping radius by considering a transit velocity for UGC 6697 of $\approx 500 \mathrm{~km} \mathrm{~s}^{-1}$.

The companion galaxy, CGCG $97087 \mathrm{~N}$, is losing gas while traveling almost perpendicular (from SW to NE) to the motion of UGC 6697, as suggested by the double tail emerging from the galaxy that connects to the gas distribution of UGC 6697. Similarly to UGC 6697, CGCG $97087 \mathrm{~N}$ also has a depleted front where we do not detect any gas emission, but differently from its massive companion, its tail is split into two and completely lacks HII regions. For UGC 6697, the integrated $\mathrm{H} \alpha$ flux in the low-velocity map is $1.16 \times 10^{-13} \mathrm{erg} \mathrm{s}^{-1} \mathrm{~cm}^{-2}$ and $5.2 \times$ $10^{-13} \mathrm{erg} \mathrm{s}^{-1} \mathrm{~cm}^{-2}$ in the high-velocity frame. CGCG $97087 \mathrm{~N}$ instead has an $\mathrm{H} \alpha$ flux as low as $6.9 \times 10^{-16} \mathrm{erg} \mathrm{s}^{-1} \mathrm{~cm}^{-2}$ and $1.23 \times 10^{-14} \mathrm{erg} \mathrm{s}^{-1} \mathrm{~cm}^{-2}$ in the low- and high-velocity maps, respectively. The sum of the fluxes of the two component is in good agreement with the independent values published by Yagi et al. (2017), obtained from the narrow-band imaging with the Subaru telescope $\left(5.8 \pm 1.1 \times 10^{-13} \mathrm{erg} \mathrm{s}^{-1} \mathrm{~cm}^{-2}\right)$.

\subsection{Velocities}

Figure 4 shows the velocities of the ionized gas, separated into low- and high-velocity components, and of the stellar component along the line of sight after subtracting the systemic velocity of UGC 6697. The velocities of CGCG 97087N are also displayed in Fig. 8. Both galaxies are seen nearly edge-on with clear evidence of a rotation curve in both the gas (first two rows) and the stars (bottom panel). The rotation curve of stars as traced by the CaII triplet is smooth and fairly symmetric in both galaxies. UGC 6697, the most massive of the two galaxies, rotates with a velocity of $\approx 250 \mathrm{~km} \mathrm{~s}^{-1}$ (at a radius of $\approx 14 \mathrm{kpc}$ ), while its smaller companion shows a rotational velocity of $\approx 100 \mathrm{~km} \mathrm{~s}^{-1}$ (at a radius of $\approx 3 \mathrm{kpc}$ ). The rotation curve appears fairly regular and disfavors a scenario in which the two galaxies have been interacting tidally. However, a mild asymmetry is present at the NW edge of the stellar component of UGC 6697. On the other hand, the interaction between the ISM and the ICM causes the gaseous component to leave UGC 6697 in the NW direction. Since the motion of the galaxy through the cluster is believed to be almost in the plane of the sky, the drag due to ram pressure does not decelerate the gas along the line of sight. In this geometry, the stripped gas would preserve its pre-stripping dynamical status. In other words, the redshift of the stripped gas would remain close to the value it had before stripping, that is, when it was attached to the stars, thus preserving memory of the stellar rotation curve.

In velocity space, the tail of UGC 6697 is divided into two substructures that are sharply separated by $\approx 200 \mathrm{~km} \mathrm{~s}^{-1}$ : the upper layer of the tail (in orange in the central map) connects to the NW edge of the stellar component and its velocity is around $250 \mathrm{~km} \mathrm{~s}^{-1}$; the lower layer of the tail (in green/blue in the central map) is at much lower velocities $\left(-100-0 \mathrm{~km} \mathrm{~s}^{-1}\right)$ and connects to the central and SE-front part of the galaxy. This difference in velocities suggests that similarly to CGCG $97087 \mathrm{~N}$, the gas stripped from UGC 6697 is also distributed along two tails that are seen almost in perfect superposition. In correspondence of the upper layer of the tail, in the low-velocity map, we trace the underlying low-velocity gas that may be associated with the lower layer of the tail. All the HII regions that we detect in the NW tail are univocally associated with the gas, with the highest velocity that connects to the NW edge of the galaxy.

The velocity dispersions of the two components are displayed in Fig. 6. Far in the NW tail, where the HII regions lies, the gas displays low-velocity dispersions with respect to 
G. Consolandi et al.: Tomography of UGC 6697, a massive galaxy falling into Abell 1367
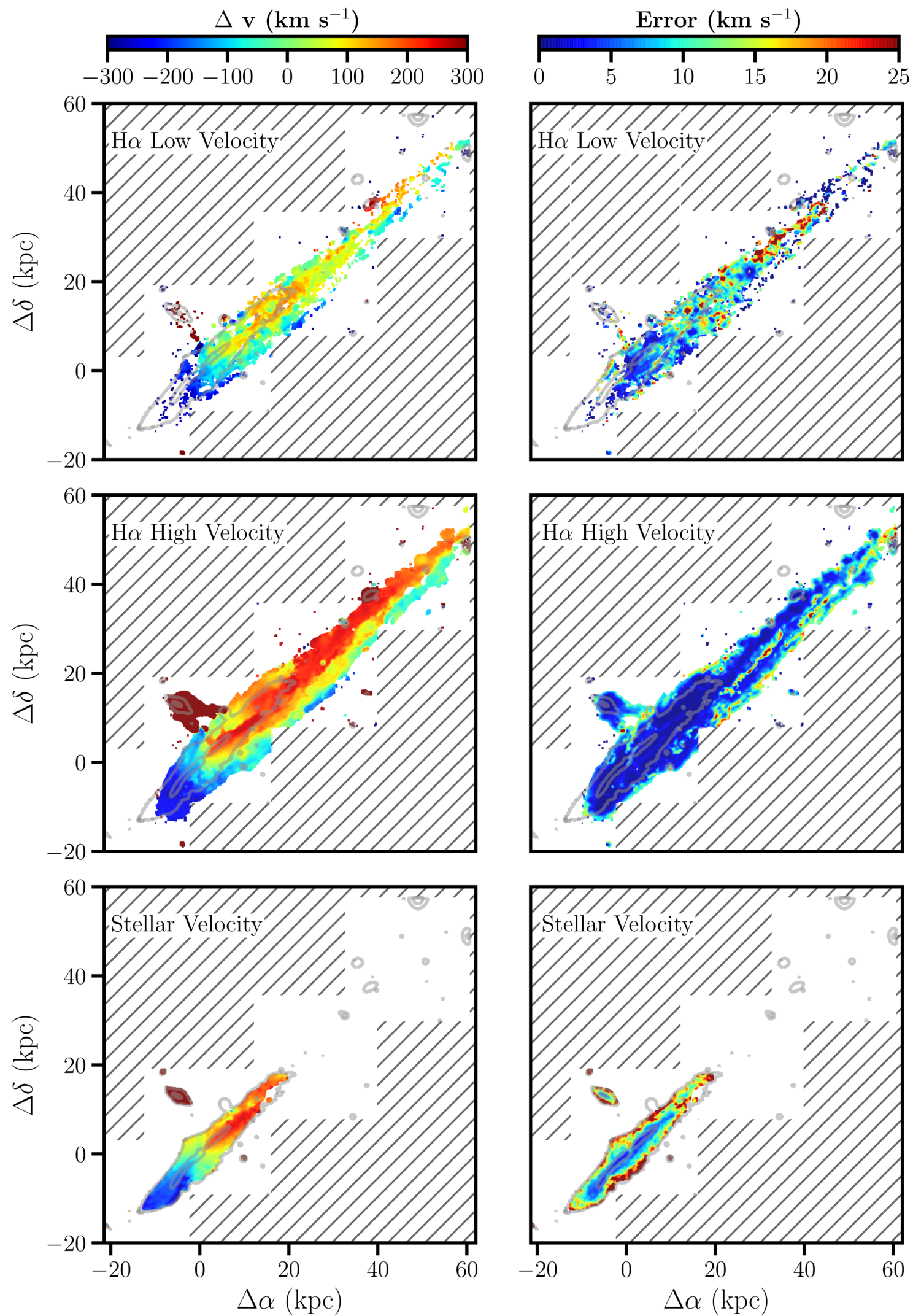

Fig. 4. MUSE field centered on UGC 6697 smoothed by $10 \times 10$ fit with the $\mathrm{H} \alpha+[\mathrm{NII}]$ lines assuming double velocity components. The first row shows the velocity field and the associated error map of the low-velocity component of the gas. In the second row, we present the velocity field and the associated error map of the high-velocity component. Finally, in the bottom row, we plot the stellar velocity map derived from the fit of the Ca II line and its associated error. The extent of the stellar continuum is given by the gray contours that represent the 2 th $\mathrm{mag}$ arcsec ${ }^{-2}$ and the 20th mag arcsec ${ }^{-2}$ isophotes in the SDSS $r$-band image reconstructed from the MUSE datacube. We note that the SE tip of the galaxy does not show any line emission. 


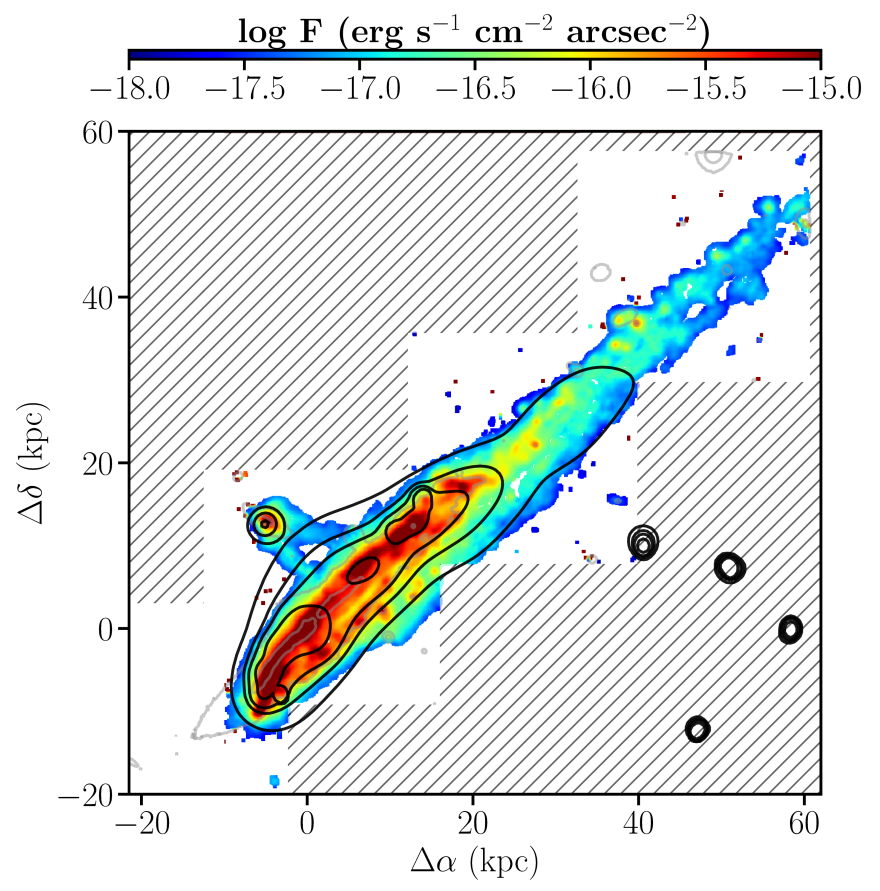

Fig. 5. Total flux distribution of $\mathrm{H} \alpha$ emission with the Chandra contours superimposed to show the region of the X-ray emitting gas. The X-ray map has been smoothed, but the X-ray front is at the same position as the $\mathrm{H} \alpha$ front. The X-ray tail appears cospatial to the $\mathrm{H} \alpha$ tail.

the surrounding gas, in contrast to the low-velocity gas, which retains higher values of the velocity dispersion. This is suggestive of more turbulent kinematics. The observed velocity dispersion of the gas outside the HII regions in the disk and in the tail is broadened up to $\approx 150 \mathrm{~km} \mathrm{~s}^{-1}$, that is, approximately five times higher than the expected value for a spiral galaxy at $z \sim 0$ (Wisnioski et al. 2015).

Figure 7 sketches our cartoon model for UGC 6697, including the presence of double velocity components. In this model, the galaxy is in fast motion through the ICM and is observed along an almost edge-on view. Its front (shaded) is already completely depleted of gas. The PSB spectral features in this region testify to an abrupt truncation of the star formation. As the line of sight approaches the central region, we detect gas at increasing velocity, following the rising rotation curve of the stars. More to the NW, most lines of sight will cross regions containing multiple velocities: those connected to the stellar rotation curve (marked 200) are blended with those containing stripped gas (marked -50) that originate in the SE and retain the kinematics of their places of origin. The spectrum (inset of Fig. 7) shows a typical double-horned profile of $\mathrm{H} \alpha$ and [NII] with separation exceeding $200 \mathrm{~km} \mathrm{~s}^{-1}$, as observed mainly in the down-stream half of UGC 6697.

Figure 8 shows the velocity field of CGCG 97087N for its stellar and gaseous component separately. For the gaseous component, we plot the high-velocity component since we detect only few spaxels in the low-velocity component, representing a negligible percentage of the flux belonging to CGCG 97087N. The map shows the gas that is dragged out of the galaxy by the ram pressure from NE to SW. Its lower rotational speed is consistent with the low mass measured by Consolandi et al. (2016), and the high recessional velocity with respect to the cluster suggests a motion through the cluster with a significant component along the line of sight.
The stellar component rotation curve is fairly smooth, with only a mild velocity gradient. Conversely, the map of the gas looks rather different. The ionized gas is stripped along two tails that are separated spatially and kinematically: the southern (S) tail and the northern $(\mathrm{N})$ tail. Along the $\mathrm{N}$ tail we detect velocities that are very similar to the velocities of the stars (between -100 and $100 \mathrm{~km} \mathrm{~s}^{-1}$ ). In contrast, the $\mathrm{S}$ tail shows a much steeper velocity gradient: the velocity of the gas from the $\mathrm{S}$ edge of the galaxy continuously decreases to $\approx-400 \mathrm{~km} \mathrm{~s}^{-1}$. This gradient is not consistent with the velocities displayed by the velocity curve of the stars. Instead, this significant deceleration of the gas could be caused by the drag induced on the ISM by the cluster ICM. In this case, however, a similar deceleration should appear in the $\mathrm{N}$ tail as well. However, we tend to exclude that the gas that we observe at $-400 \mathrm{~km} \mathrm{~s}^{-1}$ instead belongs to UGC 6697, which would support a possible direct interaction between the two galaxies. There is in fact a gap of more than $500 \mathrm{~km} \mathrm{~s}^{-1}$ between the lowest velocity in the $\mathrm{N}$ tail and the velocity of the gas that unambiguously belongs to UGC 6697 observed near the region of putative crossing. Nevertheless, the flux of both tails eventually connects to the gas distribution of UGC 6697 (see also Fig. 4), but there is no evident continuity in the velocity distributions. However, Fig. 6 shows that the dispersion in the tails of CGCG $97087 \mathrm{~N}$ seem to form a continuum with the high-velocity dispersion gas belonging to UGC 6697 in its upper periphery, suggesting a physical connection between the two galaxies.

It is therefore interesting to test the possibility that a fast hydrodynamic interaction occurred between the two galaxies, possibly enhancing the ram pressure acting on CGCG 97087N. Signal from the tails of CGCG $97087 \mathrm{~N}$ is detected from 7245 to $7647 \mathrm{~km} \mathrm{~s}^{-1}$. In absence of a precise 3D distance indicator, it is impossible to establish whether the gas trailing behind CGCG $97087 \mathrm{~N}$ is just projected on UGC 6697 (either on the foreground or on the background) or has indeed crossed the disk of the main galaxy. If projected, it would indicate that CGCG $97087 \mathrm{~N}$ is under the action of ram-pressure stripping by the ICM of Abell 1367. Conversely, gas would be stripped by the combined action of the ICM and the ISM of UGC 6697. In either case, CGCG 97087N would be a member of Abell 1367 and not a background object.

In order to find evidence of a past interaction between the two galaxies, we need to detect gas that is deeply embedded in the signal from UGC 6697. In principle, after the continuum is properly subtracted, the difference in redshifts should automatically separate the fluxes associated with the two galaxies. Unfortunately, the difference in redshift is such that the $\mathrm{H} \alpha$ emission of the tails of CGCG $97087 \mathrm{~N}$ falls at the wavelengths of the [NII] $\lambda 6583$ line of UGC 6697. Moreover, the tails cross each other at the position where the emission from UGC 6697 is at its maximum, so that the [NII] $\lambda 6583$ line outshines the faint signal of the $\mathrm{H} \alpha$ line that belongs to the tail of CGCG $97087 \mathrm{~N}$. In these regions the signal of UGC 6697 is also characterized by a double velocity component that is brighter than the signal from CGCG $97087 \mathrm{~N}$ and hampers the detection of a putative third component.

In order to overcome this problem, we used a non-parametric method to model and subtract the continuum and isolate the emission of the $\mathrm{H} \alpha$ line. In each individual spaxel, the procedure started from the first element on the wavelength dimension, and for each spectral step, it derived a redshift $(z)$ assuming that the flux $(F(\lambda))$ at each wavelength $(\lambda)$ is $[\mathrm{NII}] \lambda 6548$ that has a flux of $1 / 3$ of the flux of the second line of the [NII] at 16583. Under these assumptions, the code examined the flux at 


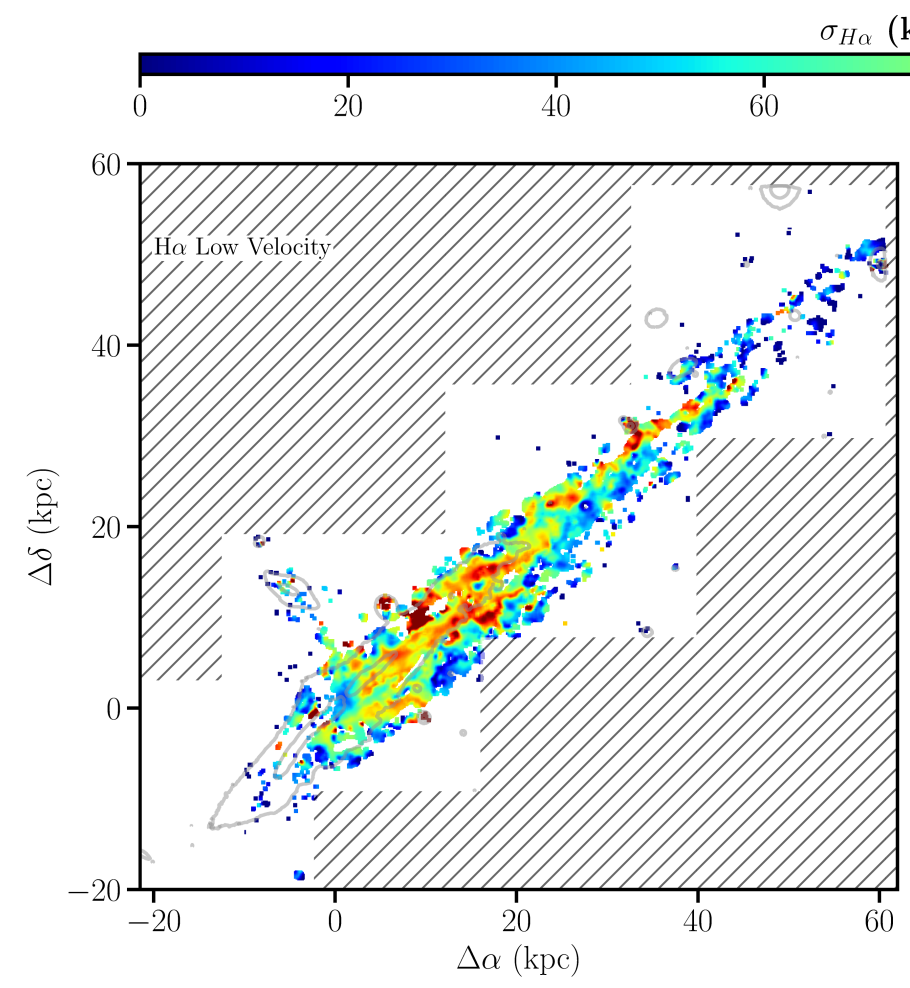

$\begin{array}{cccc} & & \\ 80 & 100 & 120 & 140\end{array}$

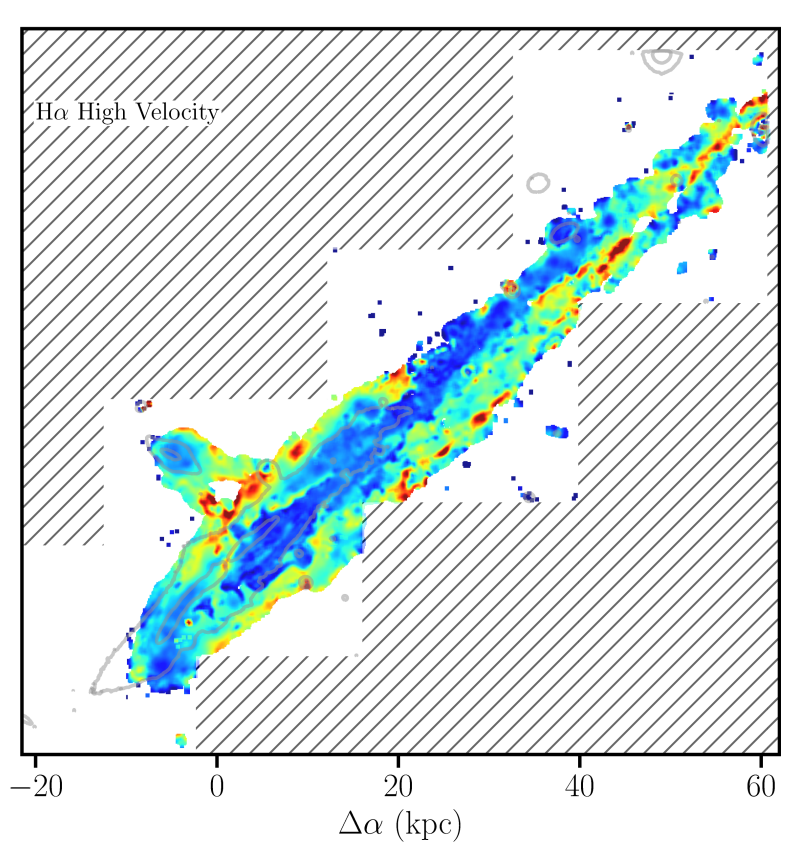

Fig. 6. Velocity dispersion map derived from the $\mathrm{H} \alpha$ line, fit with a double velocity component. In the left panel we plot the low-velocity component and in the right panel the high-velocity component. Gray contours represent the 23th and the 20th mag $\operatorname{arcsec}^{-2}$ isophotes in the SDSS $r$-band image obtained from the datacube.

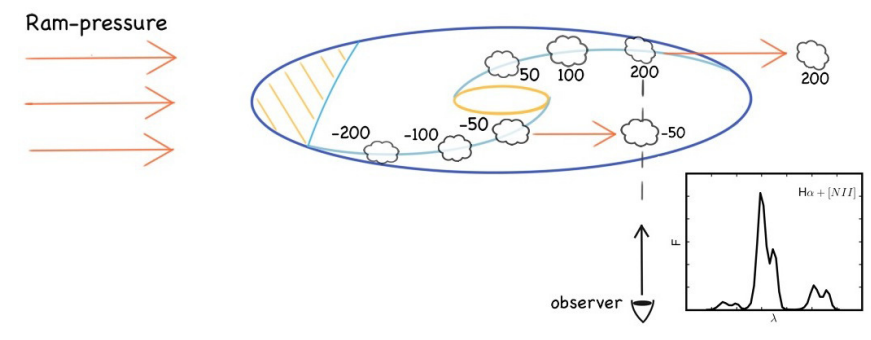

Fig. 7. Cartoon of UGC 6697 under the action of ram pressure due to its fast motion through the ICM. Its front (shaded) is already completely depleted of gas. The velocity of the gas is shown to follow the velocity curve of the stars. Velocities are given normalized to zero in the center. Some gas originally belonging to a central region has been displaced to the NW by ram pressure, retaining its original velocity $\left(-50 \mathrm{~km} \mathrm{~s}^{-1}\right)$. This cloud overlaps spatially with another gas cloud in the background arm, with a velocity of $200 \mathrm{~km} \mathrm{~s}^{-1}$. An observer who looks at the galaxy almost edge on collects a spectrum carrying multiple velocity components along the NW tail.

$\lambda_{2}=\lambda+\Delta \lambda_{[\mathrm{NII}]} \times(1+z)$, and if $F\left(\lambda_{2}\right)>3 \times F(\lambda)$, the procedure flagged emission as [NII] $\lambda 6583$. The procedure then subtracted the $F(\lambda)$, and at wavelength $\lambda_{2}$, subtracted $3 \times F(\lambda)$.

This procedure successfully recognized the [NII] lines and the subtraction was satisfactory, although not perfect, especially in the wings of the emission line. In the regions where the [NII] lines are very bright, the subtraction was performed at the 5-10\% percent level. In Fig. 9 we show the contour map of the channel at the velocity where the H $\alpha$ of CGCG 97087N is found. Surface brightness contours are shown in steps of $\times 10$ starting from $\approx 3.3 \times 10^{-19} \mathrm{erg} \mathrm{s}^{-1} \mathrm{~cm}^{-2} \operatorname{arcsec}^{-2}$. It is intriguing that the subtraction of the $[\mathrm{NII}]$ lines leaves a

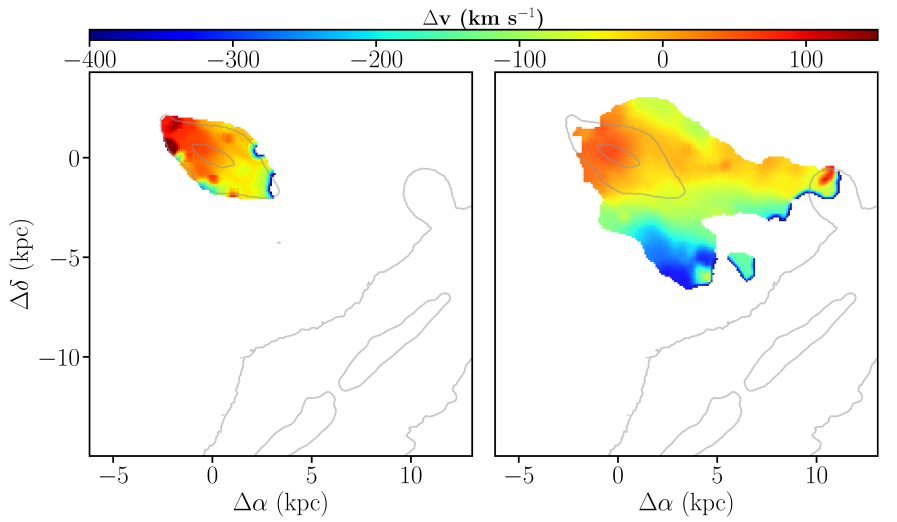

Fig. 8. Velocity maps centered on CGCG $97087 \mathrm{~N}$ of the stellar component as derived from the $\mathrm{Ca}$ II lines (left) and of the gaseous component (right) as traced by the $\mathrm{H} \alpha$. Gray contours represent the 23th and the 20th mag $\operatorname{arcsec}^{-2}$ isophotes in the SDSS $r$-band image obtained from the datacube.

faint signal that is consistent with $\mathrm{H} \alpha$ emission belonging to CGCG $97087 \mathrm{~N}$, which is elongated along the NW on the main body of UGC 6697 . The appearance of this faint but well-defined double tail that naturally connects with the emission observed outside the bright signal of UGC 6697 suggests that this embedded signal is real, and that CGCG $97087 \mathrm{~N}$ interacted hydrodynamically with UGC 6697.

In order to quantify the enhancement of the ram pressure acting on CGCG $97087 \mathrm{~N}$ through the ISM of UGC 6697, we evaluated the anchoring force of CGCG $97087 \mathrm{~N}$ as a function of the radius ( $\left.\Pi=2 \pi G \Sigma_{\text {gas }} \Sigma_{\text {stars }}\right)$ and compared it to the possible value of ram pressure due to the interaction with diffferent phases of the ISM assuming that the difference in the systemic 


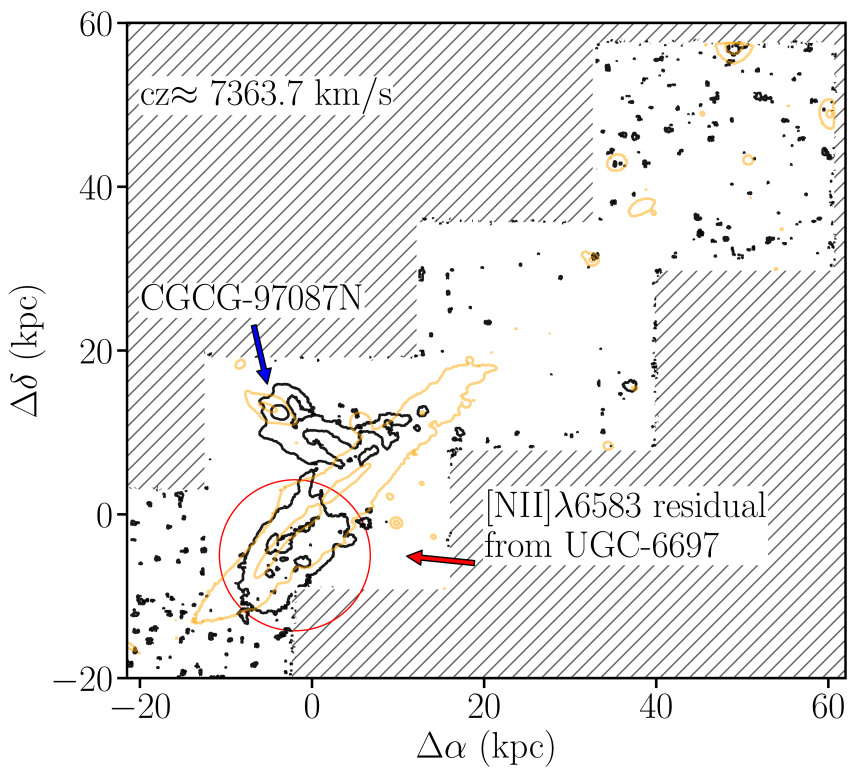

Fig. 9. Contour of the $\mathrm{H} \alpha$ flux of the tails of UGC 6697 and CGCG $97087 \mathrm{~N}$ after the [NII] emission has been isolated and subtracted. The red circle indicates the contours arising from the residual flux of the [NII] $\lambda 6583$ emitted by UGC 6697. Regions not observed are shaded in gray. Yellow contours represent the 23th and the 20th magéarcsec ${ }^{-2}$ isophotes in the SDSS $r$-band image obtained from the datacube.

velocities of the two galaxies is the relative velocity between the galaxy and the ISM. Similarly to what we did for UGC 6697, we modeled the stellar and gaseous disk with the same exponential function considering $M_{\mathrm{d} \text {,stars }}=10^{9.1} M_{\odot}$, a gas fraction of 1 , and a disk scale length of $1 \mathrm{kpc}$.

We assumed the same ICM density as for UGC 6697 and a recessional velocity of $990 \mathrm{~km} \mathrm{~s}^{-1}$. The ram pressure felt by CGCG $97087 \mathrm{~N}$ then is $P_{\text {ram }}=7.4 \times 10^{-12} \mathrm{dyn}^{-2}$. We then considered the three phases of the ISM with the greatest filling factor: the hot ionized medium $\left(n \approx 10^{-3} \mathrm{~cm}^{-3}\right.$ Draine $2011)$, which is thought to pervade much of the volume above and below the disk; the warm ionized medium $\left(n \approx 0.1 \mathrm{~cm}^{-3}\right)$, and the warm neutral medium $\left(n \approx 1 \mathrm{~cm}^{-3}\right.$ Draine 2011). When we consider a relative velocity of $870 \mathrm{~km} \mathrm{~s}^{-1}$ between CGCG $97087 \mathrm{~N}$ and UGC 6697, the ram pressure due to the dense warm ionized and neutral medium overcomes the anchoring force of CGCG $97087 \mathrm{~N}$ by orders of magnitude at all radii, possibly depleting it almost instantaneously. However, at the densities of the hot coronal ionized medium, the ram pressure would be only mildly enhanced during the short crossing time $(\approx 0.1-1$ Myr considering a gas disk scale-height of between 0.1 and $1 \mathrm{kpc}$ ), enough for the galaxy to retain part of the gas and at a stripping radius consistent with the observed radius. Hence CGCG 97087N might not have interacted with the densest phase of the ISM while crossing the disk, but possibly interacted with the less dense hot halo of UGC 6697.

\subsection{Onboard and stripped gas}

By comparing the gas properties of both the low- and the highvelocity component with the kinematics of the stars, we attempted to separate the flux and kinematics of the stripped gas (dominated by the action of ram pressure) from the gas that is possibly still onboard the galaxy. First we assumed that the gas in projection outside the stellar distribution (as traced by the CaII lines) is stripped by definition. Second, we adopted a threshold of $\Delta v=\left|v_{\text {gas }}-v_{\text {stars }}\right|=75 \mathrm{~km} \mathrm{~s}^{-1}$ in each spaxel for the gas emission to be considered either bound to the galaxy $\left(\Delta v<75 \mathrm{~km} \mathrm{~s}^{-1}\right)$ or stripped $\left(\Delta v>75 \mathrm{~km} \mathrm{~s}^{-1}\right.$; in spaxels where both velocity components have a $\Delta v$ greater than $75 \mathrm{~km} \mathrm{~s}^{-1}$ with respect to the stellar component, we consider that both belong to the stripped gas, and we take the sum of the fluxes into account and average the velocities and the velocity dispersions). This threshold was chosen to represent the quadratic sum of the maximum stellar and gas velocity dispersion expected in local disk galaxies $\left(\approx 50 \mathrm{~km} \mathrm{~s}^{-1}\right.$ Kregel et al. 2005; Wisnioski et al. 2015 ). It is worth stressing that our criterion based on the gas kinematics and geometry does not separate gas that is gravitationally bound from the gas that will be lost in the ICM. Instead, some of the gas close to the galaxy that we considered to be stripped may eventually fall back after the ram pressure becomes negligible.

Figure 10 shows the result of our attempt to separate the stripped gas from the gas that remains in the galaxy. We estimate that the flux still attached to UGC 6697 is $\approx 3.2 \times$ $10^{-13} \mathrm{erg} \mathrm{s}^{-1} \mathrm{~cm}^{-2}$, which is comparable with the stripped gas. This has a flux of $\approx 2.8 \times 10^{-13} \mathrm{erg} \mathrm{s}^{-1} \mathrm{~cm}^{-2}$. As for CGCG $97087 \mathrm{~N}$, the flux splits into $\approx 9.2 \times 10^{-15} \mathrm{erg} \mathrm{s}^{-1} \mathrm{~cm}^{-2}$ for the gas still associated with the galaxy and $\approx 3.63 \times$ $10^{-15} \mathrm{erg} \mathrm{s}^{-1} \mathrm{~cm}^{-15}$ that is stripped away. The onboard gas is associated with the brightest regions of the continuum (see contours) and with the NW side of the galaxy.

The second and third rows of Fig. 10 show the kinematics (velocity and dispersion) as traced by the $\mathrm{H} \alpha$ line. It is remarkable that this simple separation based solely on the velocity difference between stars and gas, with no further assumptions on the velocity dispersion, leads naturally to separating a component dominated by low-velocity dispersions from a component characterized by a higher average velocity dispersion. The gas considered to be onboard has a dispersion comparable to the expected values of Wisnioski et al. (2015), while the gas considered to be stripped shows a much higher velocity dispersion. The only exception is in the upper layer of the tail, where most HII regions in the tail are found. This region is characterized instead by a low-velocity dispersion (blue) similar to the gas bound to UGC 6697. Once again, the velocity dispersion map of the tails of CGCG 97087N (bottom right in Fig. 10) displays a structure that connects to the gas of UGC 6697, again suggesting a physical connection between the two galaxies.

After the stripped gas was separated from the gas that remains bound to the galaxy, we quantified the mass of ionized gas that was ripped from the galaxy. This can be estimated from the electron density by assuming a geometry for the $\mathrm{H} \alpha$ emission of the stripped gas. The electron density can be derived from the $\mathrm{H} \alpha$ luminosity using the relation

$L(\mathrm{H} \alpha)=n_{\mathrm{e}} n_{\mathrm{p}} \alpha_{\mathrm{H} \alpha}^{\mathrm{eff}} V f h v_{\mathrm{H} \alpha}$

(Osterbrock \& Ferland 2006), where $n_{\mathrm{e}}$ and $n_{\mathrm{p}}$ are the number density of electrons and protons, $\alpha_{\mathrm{H} \alpha}^{\mathrm{eff}}$ is the $\mathrm{H} \alpha$ effective recombination coefficient, $V$ is the volume of the emitting region, $f$ is the filling factor, $h$ is the Planck constant, and $v_{\mathrm{H} \alpha}$ is the frequency of the $\mathrm{H} \alpha$ emission line. Unfortunately, this estimate is affected by the high degree of uncertainty in some parameters. In particular, the exact geometry of the tail is unknown, and we can only approximate its structure assuming that the emitting region has a cylindrical shape of diameter equal to the height of the galaxy and length of $\approx 90 \mathrm{kpc}$. Because the morphology of the tail indicates a filamentary distribution that contains highdensity clumps and compact knots, a filling factor lower than 1 
G. Consolandi et al.: Tomography of UGC 6697, a massive galaxy falling into Abell 1367
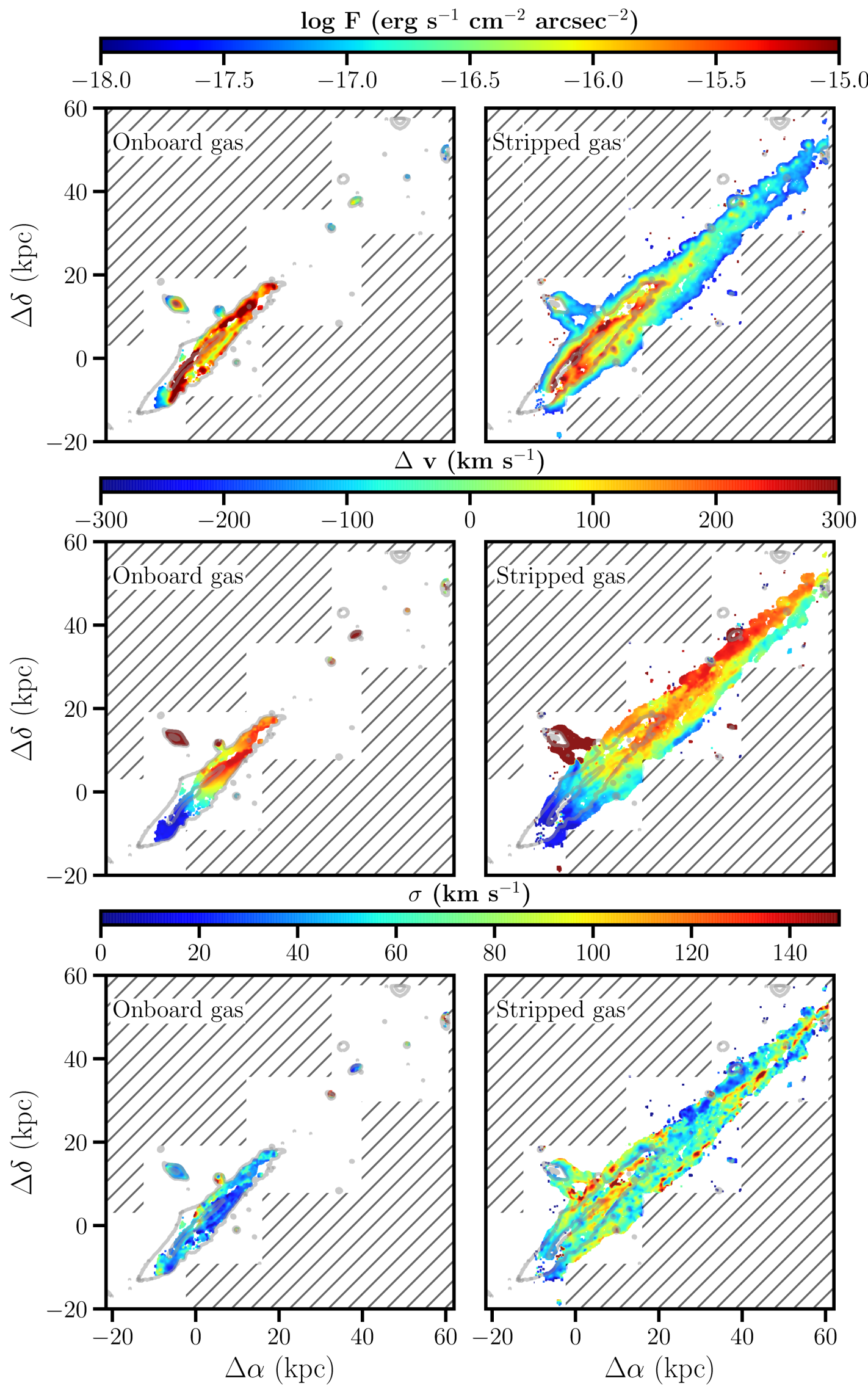

Fig. 10. MUSE field centered on UGC 6697 smoothed by $10 \times 10$ pixels fit with two sets of the $\mathrm{H} \alpha+[\mathrm{NII}]$ lines with different velocities along the line of sight. After adopting a $\delta v$ threshold of $75 \mathrm{~km} \mathrm{~s}^{-1}$ between gas and stars to distinguish the gas emission that is still bound to the galaxy and the stripped gas emission, we separate the onboard gas (left column) and the stripped gas (right column). The flux, velocity, and velocity dispersion maps are displayed from top to bottom, and the gray contours and the cross in each panel are the same as in previous figures. 
was assumed. In particular, we assumed $f=0.1$, consistent with previous works, which generally considered $0.05<f<$ 0.1 (Fossati et al. 2016; Boselli et al. 2016). Moreover, we assumed that the gas is fully ionized, thus implying $n_{\mathrm{e}}=n_{\mathrm{p}}$ and $\alpha_{\mathrm{H} \alpha}^{\mathrm{eff}}=1.17 \times 10^{-13} \mathrm{~cm}^{3} \mathrm{~s}^{-1}$ (Osterbrock \& Ferland 2006). When we assume a distance of $94.8 \mathrm{Mpc}$, the resulting electron number density is on the order of $0.6 \mathrm{~cm}^{-3}$, which is in agreement with the density estimates by Yagi et al. (2007) and Fossati et al. (2012, 2016). The implied mass of ionized gas dragged outside the galaxy is $\approx 10^{9.1} M_{\odot}$, and we estimate its recombination time to be $\approx 0.2$ Myr using

$\tau_{r}=\frac{1}{n_{\mathrm{e}} \alpha_{\mathrm{A}}}$

where $\alpha_{\mathrm{A}}$ is the total recombination coefficient equal to $4.2 \times$ $10^{-13} \mathrm{~cm}^{3} \mathrm{~s}^{-1}$ (Osterbrock \& Ferland 2006). The estimated stripped mass is consistent with the gas mass retained at radii $r \gtrsim$ $10 \mathrm{kpc}$ (the observed stripping radius) in a $10^{10.5} M_{\odot}$ disk galaxy modeled with an exponential function in a similar way as in the first paragraph of Sect. 5 .

By considering the projected separation between the stripped gas at lowest velocity in the NW tail and the region of the galaxy where stars have a similar velocity, we estimated a timescale for gas stripping. Assuming that the gas is instantaneously accelerated to the cluster velocity, we estimated that these plumes were stripped at least $\approx 60-120 \mathrm{Myr}$ ago, assuming that the galaxy travels at $\approx 500-1000 \mathrm{~km} \mathrm{~s}^{-1}$ in accordance with the upper limit for its velocity estimated by Sun \& Vikhlinin (2005). Comparing this stripping timescale to the recombination time, we conclude that some excitation mechanisms is necessary to keep the gas of the tail ionized.

Moreover, in the model proposed by Nulsen (1982) and Sun \& Vikhlinin (2005), given the orientation and geometry of the impact with the ICM of UGC 6697, mechanism such as viscous stripping and Kelvin-Helmholtz instabilities are thought to dominate the stripping of the gaseous component. Considering the density of the ICM $\rho_{\text {ICM }}=4.5 \times 10^{-4} \mathrm{~cm}^{-3}$ (Sun \& Vikhlinin 2005 ), we can evaluate the time necessary to strip $\approx 10^{9.1} M_{\odot}$ of ionized gas by Kelvin-Helmholtz instabilities if the galaxy travels through the ICM at $\approx 500-1000 \mathrm{~km} \mathrm{~s}^{-1}$ using

$\dot{M}_{\mathrm{K}-\mathrm{H}} \approx \pi r^{2} \rho_{\mathrm{ICM}} v_{\mathrm{gal}}$

(Nulsen 1982). The mass of ionized gas in the tail can therefore easily be stripped within $\approx 7-14 \times 10^{7} \mathrm{yr}$, which is consistent with the previous estimate of $\approx 60-120 \mathrm{Myr}$.

\section{Excitation mechanisms}

To constrain the ionizing processes that are acting on the gas of the tail, we considered the ratios of the emission lines. In particular, we focused on the BPT diagnostic diagram (Baldwin et al. 1981), considering lines that fall within the MUSE spectral window: $\mathrm{H} \beta \lambda 4861$, [OIII] $\lambda 5007$, [OI] $\lambda 6300$, and [NII] $\lambda 6584$. The double velocity component fit to each line kept the velocity and velocity dispersion of the two velocity components fixed to the values evaluated during the fit of the $\mathrm{H} \alpha$ line. This ensures that all lines were fit with consistent kinematics fixed by the best resolved and brightest line available. The maps showing the distribution of the fluxes of all emission lines that we fit are displayed in Fig. 11. These fluxes are the sum of the two velocity components. The [SII] lines were not considered because they fall at the wavelength of a strong sky emission line, preventing a robust flux determination.
Emission lines are detected along the main body of the galaxy, while in the outer tail, the distributions differ significantly. Hydrogen $(\mathrm{H} \alpha$ and $\mathrm{H} \beta)$ is found in both the diffuse gas and HII regions along the whole extent of the tail. The [OIII] $\lambda 5007$ line is preferentially found in the HII regions of the tail, and only little flux belongs to diffuse gas emission; conversely, [OI] $\lambda 6300$ is fainter and appears less important in HII regions.

Furthermore, following the same criteria that we adopted to separate the onboard and stripped $\mathrm{H} \alpha$, we split each line into an onboard and a stripped component.

\subsection{Line ratio maps}

Line ratio maps are displayed in Figs. 12 and 13 for the onboard and stripped gas, respectively. The top row of Fig. 12 shows the $[\mathrm{NII}] \lambda 6584 / \mathrm{H} \alpha$ maps. This ratio maintains modest values $(\sim 0.2-$ 0.3 ) in the main body of CGCG $97087 \mathrm{~N}$, while in the central region of UGC 6697, the ratio is slightly enhanced with respect to the rest of the galaxy. The value also varies significantly in the CGCG $97087 \mathrm{~N}$ tails, where it is greatly enhanced, and in the tail of UGC 6697, where it drops to values lower than 0.2. This ratio is enhanced by shocks (Kewley et al. 2001; Allen et al. 2008), and in our map of the onboard gas, the highest values are found along an elongated central region of the main body of UGC 6697 and at the surface of contact where the tails of CGCG 97087N point. In the stripped gas maps, the highest values are in the wings and in the tails that connect CGCG $97087 \mathrm{~N}$ to the galaxy. The gas characterized by high values of the $[\mathrm{NII}] \lambda 6584 / \mathrm{H} \alpha$ ratio connects and mixes with the gas that belongs to UGC 6697 , suggesting once again a physical connection between the gas of the two galaxies. In contrast, in the tail of UGC 6697, the ratio drops to values below 0.2 .

The main difference between the gas that we consider onboard to the galaxy and the gas stripped in the tails is visible in the $[\mathrm{OI}] \lambda 6300 / \mathrm{H} \alpha$ map (second row of Fig. 12), another indicator of the presence of shocked gas. The gas belonging to the main body of both galaxies and to HII regions shows a very low ratio. In contrast, the gas outside the two galaxies is characterized by much higher values, reaching the maximum in the two wings of CGCG 97087N and in the surface connecting to its tails. Similarly to what has been observed in the $[\mathrm{NII}] \lambda 6584 / \mathrm{H} \alpha$ map, this strongly indicates hydrodynamic interactions between the two systems.

The [OIII] $\lambda 5007 / \mathrm{H} \beta$ map is shown in the first row of Fig. 13. The highest values of the ratio in both maps are found preferentially in regions associated with the bright HII regions that are visible both in the $\mathrm{H} \alpha$ distribution and in the $r$-band image in the south and in the NW tail. This is consistent with a higher ionization factor (assuming a constant metallicity in the stripped gas) in these regions with respect to the surrounding gas. The main body of both UGC 6697 and CGCG $97087 \mathrm{~N}$ shows onboard components with moderate [OIII] $\lambda 5007 / \mathrm{H} \beta$ values, with the exception of the peripheral layer of UGC 6697, where the HII regions lie. This is also true for the SE edge, in correspondence with the sharp cut in the ionized gas distribution that might be associated with the front of the galaxy where the ISM impacts the hot ICM. In this case, we probably view an enhanced star formation activity that is due to the compression of the gas in the front.

Finally, we show the $\mathrm{H} \alpha / \mathrm{H} \beta$ map in the bottom row of Fig. 13. This ratio is commonly used to infer dust extinction, assuming an intrinsic ratio of 2.86 from case $B$ recombination at $T=10^{4} \mathrm{~K}$ (Osterbrock 1989). In the onboard map, the main 
G. Consolandi et al.: Tomography of UGC 6697, a massive galaxy falling into Abell 1367

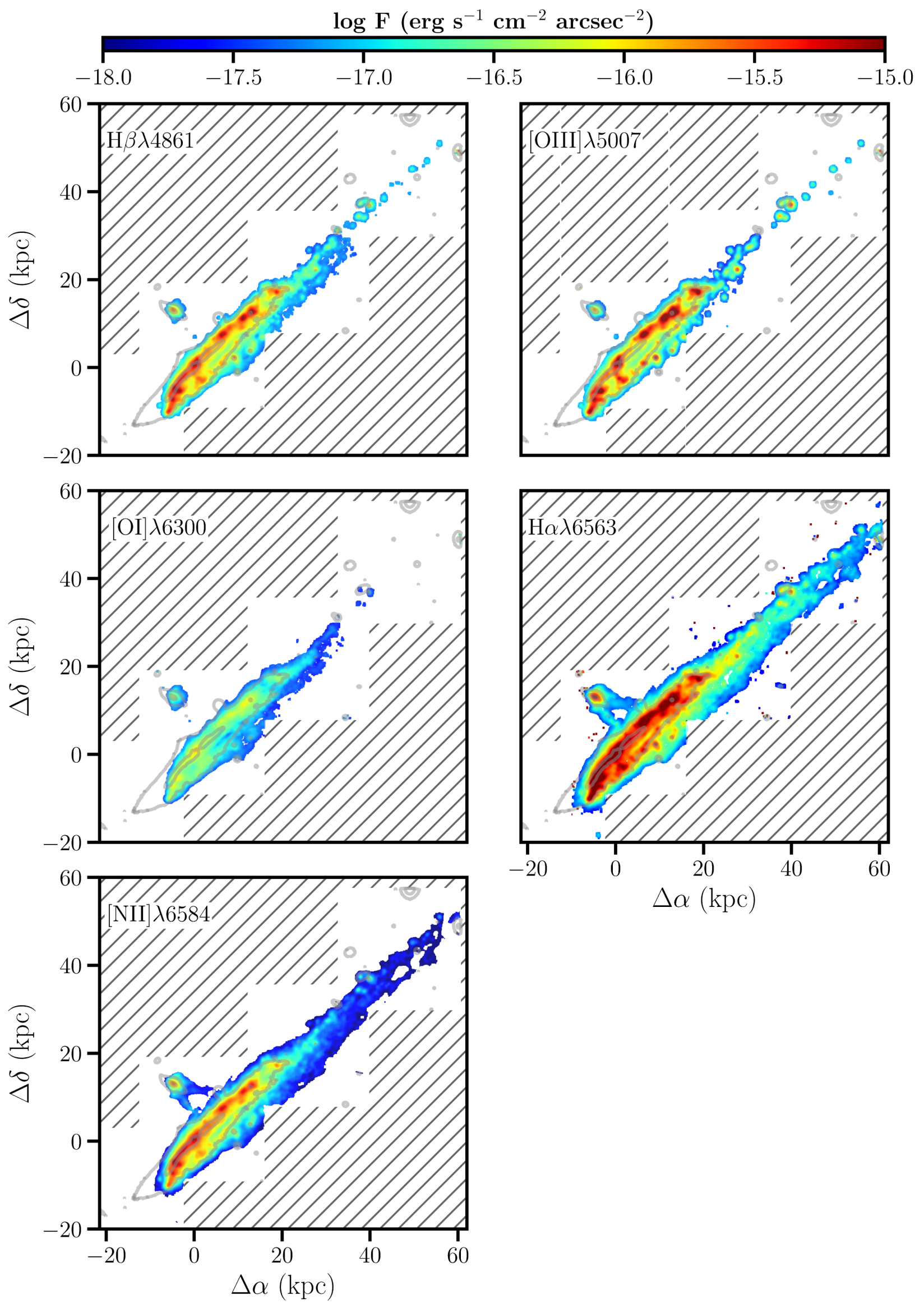

Fig. 11. Emission line maps of UGC 6697. Panels are sorted by increasing wavelength from left to right and from top to bottom. The solid contours represents the 23th and the 20th mag arcsec ${ }^{-2}$ isophotes in the SDSS $r$-band image obtained from the datacube. Areas not mapped by the MUSE mosaic are shaded in gray. 

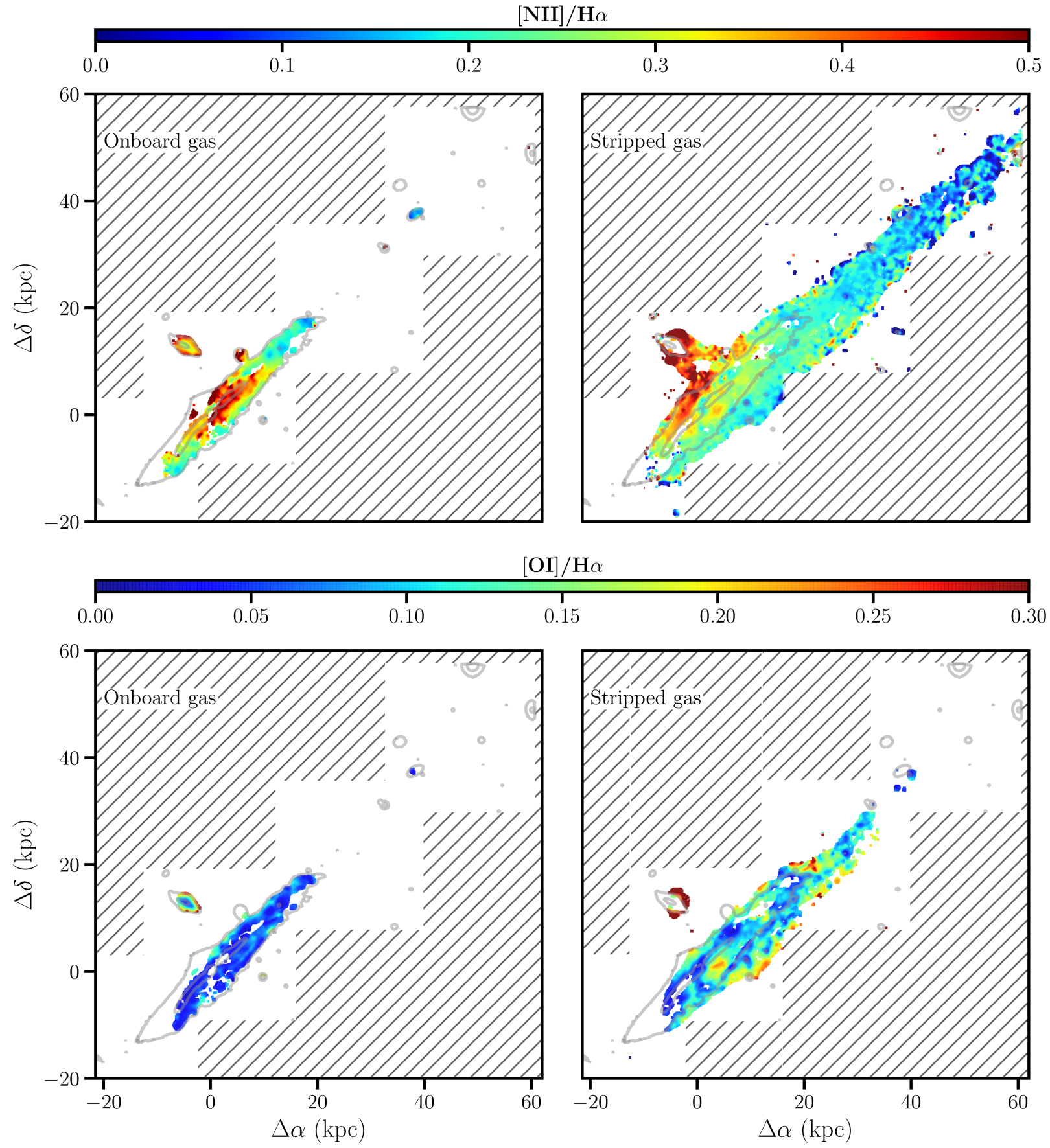

Fig. 12. Maps of line ratios for the onboard (left) and stripped (right) components. From top to bottom, we plot the [NII] $26584 / \mathrm{H} \alpha$ and $[\mathrm{OI}] \lambda 6300 / \mathrm{H} \alpha$ maps, respectively. Contours and gray shaded areas are the same as in previous figures.

body of UGC 6697 displays overall ratios that are consistent with being absorbed by the presence of dust. Conversely, the stripped gas is in line with the theoretical value of the ratio. In addition, the main body of CGCG $97087 \mathrm{~N}$ is characterized by higher ratio values $(\approx 4)$ consistent with a mild obscuration by dust. We stress that UGC 6697 also has higher values of $\mathrm{H} \alpha / \mathrm{H} \beta$ where the continuum is at a maximum, while in the tail, the ratio settles around the theoretical value. The presence of dust along the line of sight is not surprising in the disk of edge-on spiral galaxies, but we refrain from interpreting the values in the tails because other ionization mechanisms are at play, and the assumption of an intrinsic ratio of 2.86 may not hold true in these regions.

\subsection{Spatially resolved BPT diagrams}

Next we build spatially resolved BPT diagrams for the onboard and stripped gas, separately. Unfortunately, the required simultaneous detection of four emission lines in each spaxel limits the analysis to the brightest spaxels, and we cannot use the BPT diagnostic for the whole extent of the tail.

In the left panels of Fig. 14 we show the BPT diagrams based on the $\mathrm{H} \beta \lambda 4861$, [OIII] $\lambda 5007, \mathrm{H} \alpha \lambda 6563$, and [NII] $\lambda 6684$ emission lines of the onboard and stripped component, respectively. Resolution elements are color-coded according to their minimum distance from the Kauffmann et al. (2003) curve that 

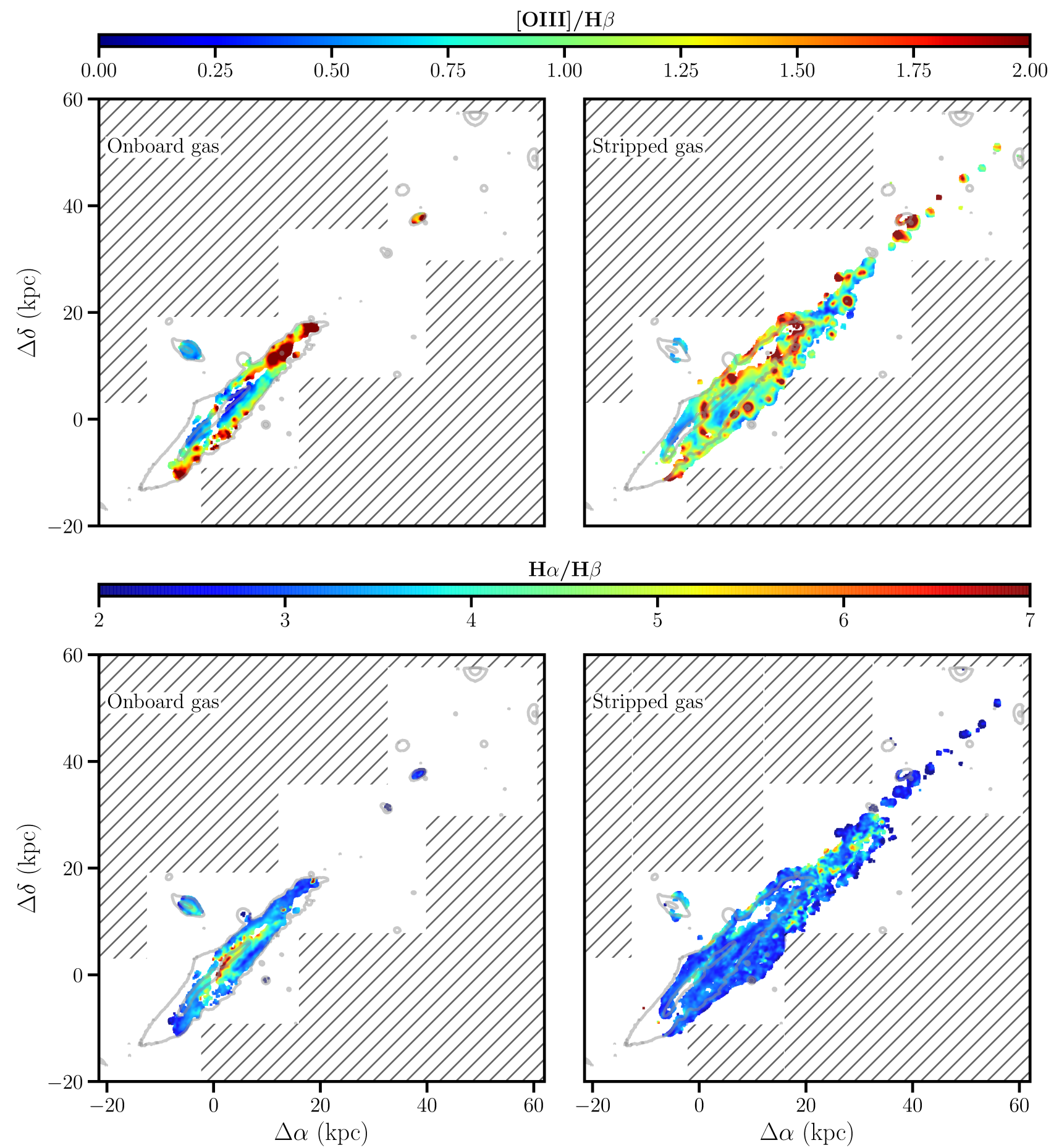

$\mathbf{H} \alpha / \mathbf{H} \beta$
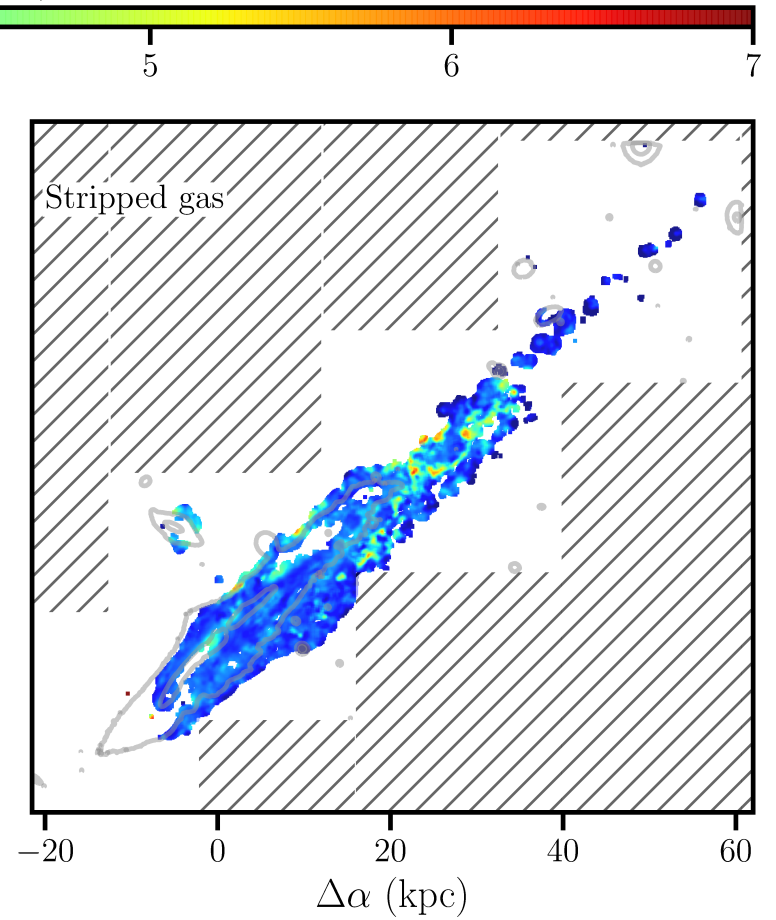

Fig. 13. Same as Fig. 12, but for the $[\mathrm{OIII}] \lambda 5007 / \mathrm{H} \beta$ and $\mathrm{H} \alpha / \mathrm{H} \beta$ maps, respectively.

separates the regions of the plane associated with photoionization from the regions associated with other ionization processes (e.g., AGN and shocks). For each BPT panel, we display on the right the map color-coded in the same way as the associated BPT digram in order to locate which region of the galaxy contributes to which points in the BPT digram. In both panels, we overplot the three different photoionization models at different metallicities $\left(0.2,0.4\right.$, and $\left.1 Z_{\odot}\right)$ from Kewley et al. (2001). The contours are obtained from a sample of nuclear spectra of SDSS galaxies in the redshift range $0.01<z<0.1$ and with stellar masses $M_{*}=10^{9}-10^{11} M_{\odot}$. In the onboard component, our data are mainly distributed along the HII region wing of the BPT: the main body of UGC 6697 and the main body of CGCG $97087 \mathrm{~N}$ are both characterized by typical ratios for gas that is photoionized by stars (the few blue points in the BPT of the onboard component are not from UGC 6697, but from the small galaxy at $x \approx 40 \mathrm{kpc} y \approx 40 \mathrm{kpc}$ ). The spaxels associated with the diffuse gas outside the galaxy (bottom panels of Fig. 14) instead exhibit a lower [OIII] $\lambda 5007 / \mathrm{H} \beta$ ratio than the HII regions inside the galaxy. This deviation from the HII region wing of the BPT can be interpreted as an effect of the lower metallicity of the peripherical gas of the galaxy. The external gas is indeed less 


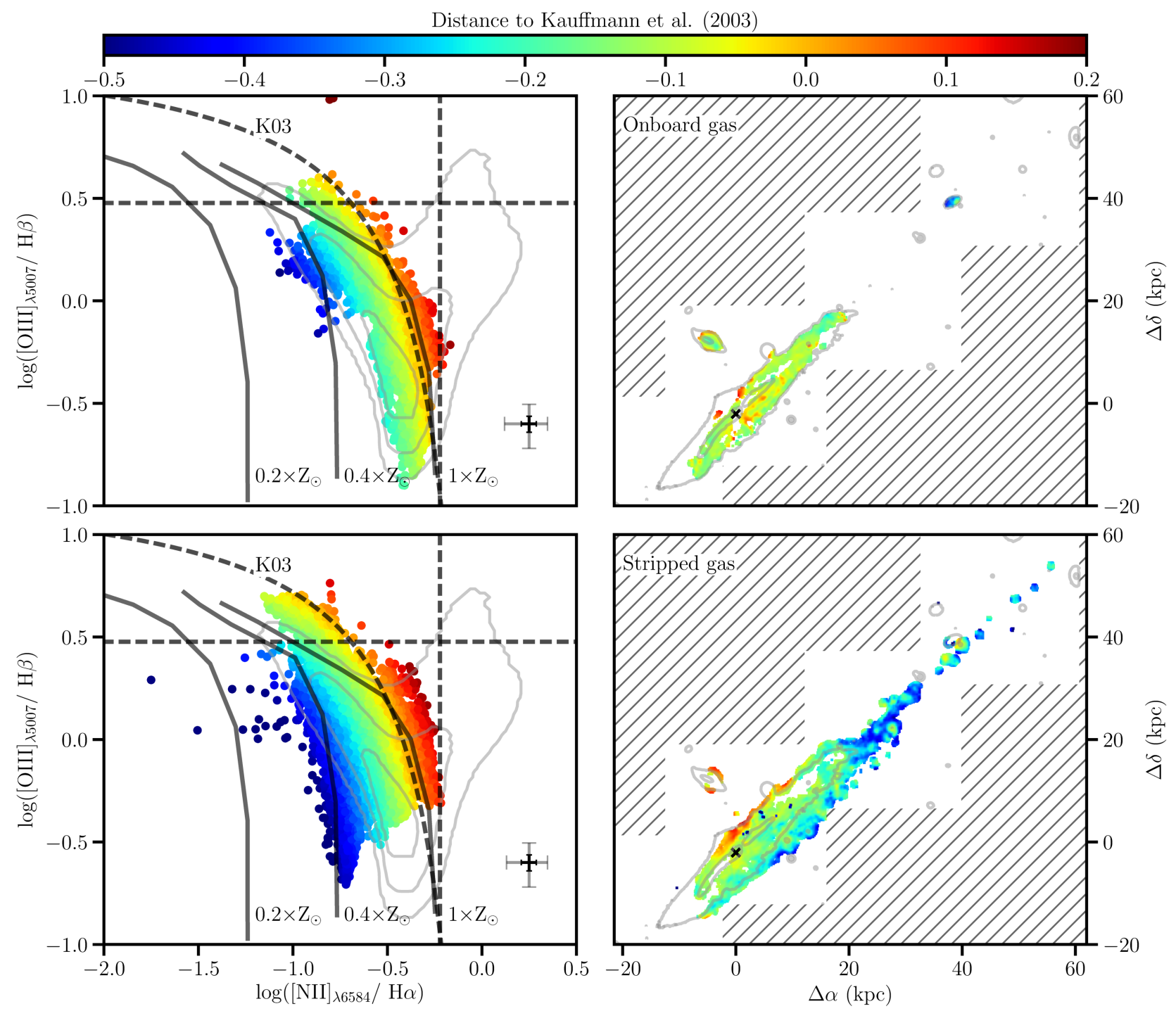

Fig. 14. Top left: BPT diagram of the onboard component of UGC 6697. The dashed curve separates AGN from HII regions and is adopted from Kauffmann et al. (2003, K03). Data are color-coded according to their minimum distance to the K03 curve. The black and gray crosses indicate the typical error of the ratio of lines with an $S / N \approx 15$ and $S / N \approx 5$, respectively. At higher $\mathrm{S} / \mathrm{N}$, the error becomes comparable to the dot size. Thick solid lines show the three different photoionization models at different metallicities $\left(0.2,0.4\right.$, and $\left.1 Z_{\odot}\right)$ by Kewley et al. (2001). The gray contours are obtained from a random sample of nuclear spectra of SDSS galaxies in the redshift range $0.01-0.1$ and with masses from $10^{9}$ and $10^{11} M_{\odot}$. Right: map of UGC 6697 of the spaxel contributing to the BPT of the onboard component, color-coded as in the left panel. The black cross indicates the center of the galaxy, while regions of the sky not mapped by the MUSE mosaic are shaded in gray. Bottom: same as in the top panels, but for the stripped component of the gas.

tightly bound to the galaxy and is stripped more easily. The photoionization model with $Z=0.4 Z_{\odot}$ of Kewley et al. (2001) falls perfectly within our data. The (red) points in the BPT that lie directly to the right of the curve of Kauffmann et al. (2003) are associated with the wings of CGCG $97087 \mathrm{~N}$ and with the upper layer of UGC 6697, where the tails of CGCG 97087N intersect UGC 6697.

In Fig. 15, we plot from top to bottom the BPT diagnostics based on $\mathrm{H} \beta \lambda 4861$, [OIII] $\lambda 5007, \mathrm{H} \alpha \lambda 6563$, and [OI] $\lambda 6300$ for the onboard and stripped component of the gas, respectively. Points and maps are color-coded according to the minimum distance from the Kewley et al. (2001) curve that limits the region consistent with photoionization. We overplot five curves that indicate the relative contribution of shocks to the ionization of the gas (Rich et al. 2011). The contours are again obtained from the same sample of nuclear spectra of SDSS galaxies previously described. In the top panel, the onboard gas is distributed mainly in the photoionization region of the diagram, and the only points crossing the limit of Kewley et al. (2001; dashed curve) are associated with the outer parts of CGCG 97087N and with peripheral regions of UGC 6697. A clear difference is observed instead in the diagram of the bottom panel: the majority of the points falls in the region to the right of the limit of photoionization of Kewley et al. (2001).

According to the models of Rich et al. (2011), roughly half of the spaxels are consistent with $70 \%$ of the ionization coming from shocks. The regions with the highest contribution from shocks (up to $100 \%$ ) are associated with the diffuse gas at the periphery of the galaxy, while the main body of both galaxies appears to be consistently photoionized by stars.

Figures 14 and 15 consistently show that the tails of CGCG $97087 \mathrm{~N}$ and the gas of UGC 6697 to which they connect is shocked (enhanced [NII] $\lambda 6684 / \mathrm{H} \alpha$ and $[\mathrm{OI}] \lambda 6300 / \mathrm{H} \alpha$ ). In the tail of UGC 6697 , the gas is characterized by the lowest values of [NII] $\lambda 6684 / \mathrm{H} \alpha$, possibly because of its low metallicity, but the high $[\mathrm{OI}] \lambda 6300 / \mathrm{H} \alpha$ also suggests that this gas is 

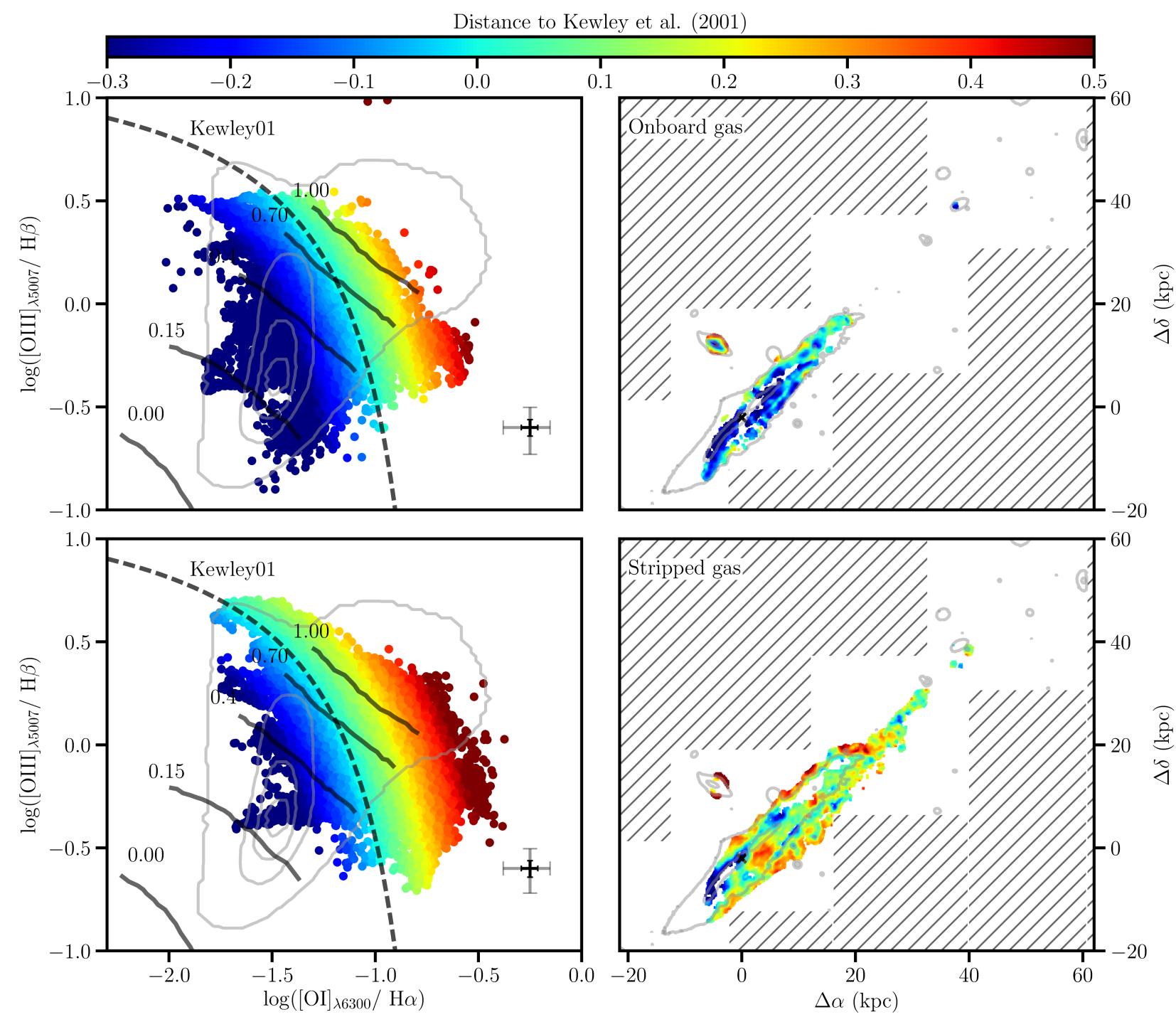

Fig. 15. Top Left: BPT diagram of the onboard component of UGC 6697 using the [OI] to $\mathrm{H} \alpha$ ratio. The dashed curve separates AGN from HII regions and is adopted from Kewley et al. (2001, kewley01). Data are color-coded according to their minimum distance to the Kewley01 curve. The black and gray crosses indicate the typical error of the ratio of lines with an $S / N \approx 15$ and $S / N \approx 5$, respectively. At higher S/N, the error becomes comparable to the dot size. Thick solid lines show the five different shock models by Rich et al. (2011), indicating that five different fractions (from 0 to 1 ) of $\mathrm{H} \alpha$ flux contributed by shocks. The gray contours are obtained from a random sample of nuclear spectra of SDSS galaxies in the redshift range 0.01-0.1 and with masses from $10^{9}$ and $10^{11} M_{\odot}$. Right: map of UGC 6697 of the spaxel contributing to the BPT of the onboard component, color-coded as in the left panel. The black cross indicates the center of the galaxy, while regions of the sky not mapped by the MUSE mosaic are shaded in gray. Bottom: same as in the top panels, but for the stripped component of the gas.

shocked and turbulent. Hence, all stripped gas shows some evidence of shocks that contribute to the ionization. The fact that the strongest indications are found in the tails of CGCG 97087N and in the gas connecting the two galaxies indicates a physical interaction. Similar conclusions were also drawn from the distribution of the velocity dispersion in these regions both in the single and in the double velocity component fit.

\section{HII regions}

Compact knots of star formation lie outside the stellar disk along the northern and to a lesser extent the southern periphery of the tail. Gas in and around these HII region is characterized by lower velocity dispersions than the surrounding medium. It is therefore interesting to study the properties of these compact knots in more detail. First, by visual inspecting the map of the [OIII] $\lambda 5007$ (the faintest line of the BPT), we selected the compact knots and extracted line fluxes in circular apertures, along with the average velocity and velocity dispersion of the $\mathrm{H} \alpha$ line. In order to test whether these compact knots are also associated with continuum emission, we collapsed the MUSE cube on the lambda dimension and searched for counterparts by visual inspection.

Moreover, we covered the brightest regions of the main body of UGC 6697 and of CGCG $97087 \mathrm{~N}$ with seven circular apertures in order to characterize the properties of the starforming regions found inside the galaxy with respect to the extra-disk knots. We measured the metallicity of the O3N2 ratio with the relation calibrated by Curti et al. (2017). The extinction was computed from the theoretical value of the $\mathrm{H} \alpha / \mathrm{H} \beta$ ratio considering the selective extinction of $\mathrm{H} \alpha$ relative to $\mathrm{H} \beta$ as $f\left(\mathrm{H}_{\alpha}\right)=-0.297$ evaluated from the Galactic extinction law of Cardelli et al. (1989). In Fig. 16 we show the BPT diagram of the HII regions identified by visual inspection. All regions appear to be normal star-forming clumps in the diagram, and we do not detect any remarkable difference between the HII regions in the main body of the galaxies and the external regions. 

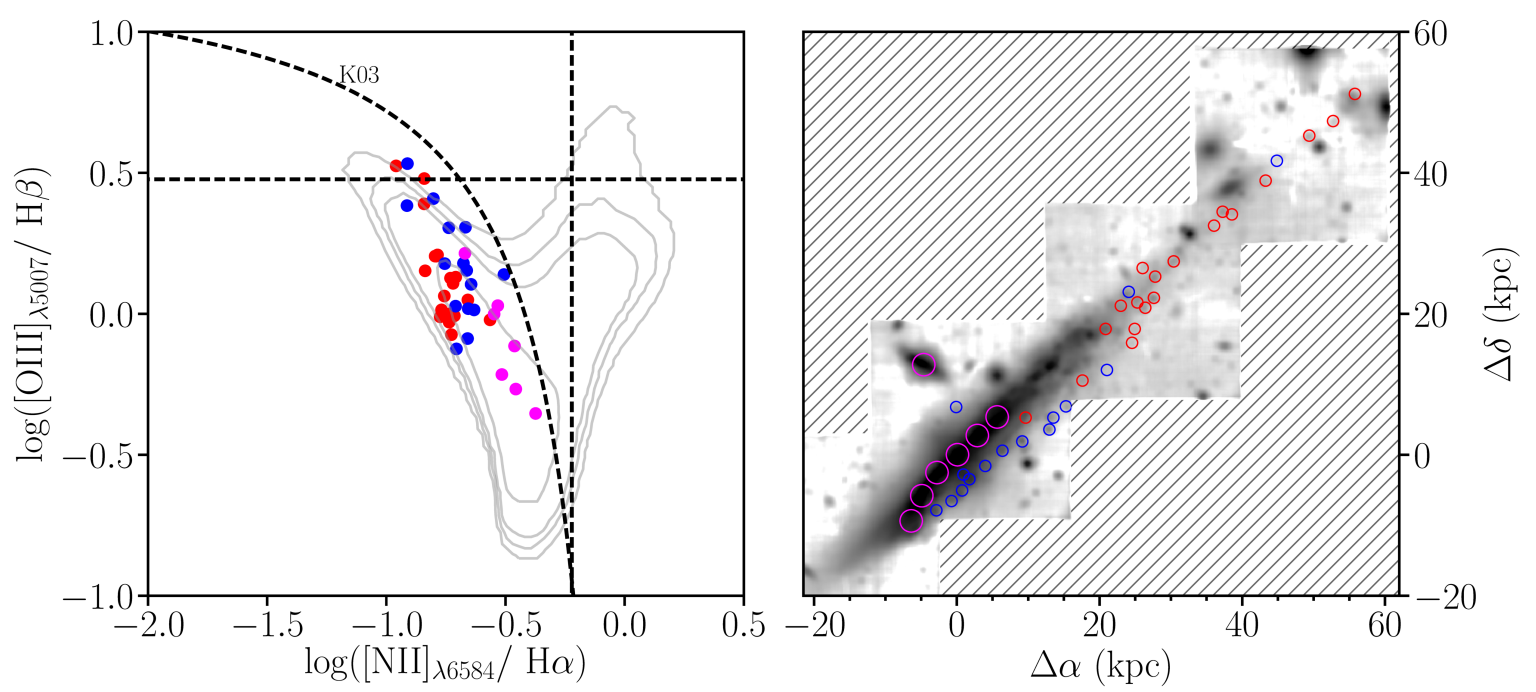

Fig. 16. Left: BPT diagram of HII regions selected by visual inspection of the [OIII] $\lambda 5007$ map. Right: the collapsed median cube smoothed $10 \times 10$ pixels with circles indicate the position of the selected HII regions. HII regions that are part of the main body of galaxies are circled in magenta, while HII regions outside the bright disk of the galaxy are circled in blue $\left(v-v_{\mathrm{sys}}<100 \mathrm{~km} \mathrm{~s}^{-1}\right)$ and red $\left(v-v_{\mathrm{sys}}>100 \mathrm{~km} \mathrm{~s}{ }^{-1}\right)$. We note that by this division, HII regions that lie outside the galaxy are neatly distributed along two separate trails.

Furthermore, no remarkable differences between the extradisk HII regions and the galactic HII regions are found in metallicity and extinction. The gas ionized inside the disk has similar metallicities compared to the gas outside the galaxy. In the disk, the average metallicity $(\langle 12+\log (\mathrm{O} / \mathrm{H})\rangle \approx 8.6)$ is close to solar, similarly to the HII regions outside the galaxy, with a metallicity between 8.4 and 8.6, in agreement with the values extracted by Gavazzi et al. (2001). Extinction is similarly consistent in both the onboard and stripped HII regions, and it is almost negligible $\left(\left\langle A_{v}\right\rangle=0.4 \mathrm{mag}\right)$.

In the velocity maps of $\mathrm{H} \alpha$, most of the HII regions in the back tail are found at velocities higher than $100 \mathrm{~km} \mathrm{~s}^{-1}$. When we separate all HII regions with such velocities from the remaining regions, we obtain the separation of red and blue symbols in Fig. 16. Considering the distribution of compact knots circled in blue and red separately, we note that these two are neatly positioned along two parallel paths running along the northern and southern boundary of the trailing gas. This suggests that there are two preferential streams along which star formation can occur in the stripped gas, which we observe in superposition because of projection effects. This evidence indicates a physical mechanism that is able to funnel the gas along preferential tracks. An effect like this has been proposed by Dursi \& Pfrommer (2008) as due to cluster magnetic field draping.

\section{Discussion and conclusions}

In this section we present a comprehensive picture of the turbulent life of UGC 6697 in the cluster, which we discuss in the context of previous environmental studies.

\subsection{The process that drives the stripping}

As discussed in Sect. 2, UGC 6697 has been under investigation since the early works of Gavazzi (1978) and until the recent deep data of Subaru (Yagi et al. 2017) and present MUSE observations. Throughout the years, hydrodynamic interactions between the ISM ad the ICM have been invoked as the main factor for the asymmetries in the young stellar distribution (i.e., in jellyfish galaxies, Poggianti et al. 2016) and in the gaseous distribution of UGC 6697.
Mainly because of the complex kinematics in the gaseous component of the galaxy, Gavazzi et al. (2001) proposed a different scenario in which UGC 6697 is a system of two merging galaxies. We tend to exclude this possibility since we can ascribe the complex gas kinematics to the superposition of emitting gas with different velocities along the line of sight while it is stripped from the SE and dragged to the NW (see Fig. 7). In the maps of the stellar kinematics of UGC 6697, we do not find evidence of two kinematically separated systems. Hence we suggest that UGC 6697 is a single massive $\left(v_{\text {rot }} \sim 250 \mathrm{~km} \mathrm{~s}^{-1}\right)$ edge-on spiral galaxy that shows the hydrodynamic interaction with the ICM.

Taking advantage of the exquisite MUSE observations, we have indeed measured and separated the gas emission of the two different components observed along the line of sight. Comparing the velocity of gas and stars, we separated the ionized gas into a stripped component and a component that is still bound to the galaxy. The gas still onboard the galaxy has low velocity dispersions $\left(\leq 50 \mathrm{~km} \mathrm{~s}^{-1}\right)$, while the stripped gas is characterized by higher velocity dispersions $\left(60-100 \mathrm{~km} \mathrm{~s}^{-1}\right)$, with the exception of some locations that harbor HII regions. This indicates that the stripped gas is characterized by turbulent motions and shocks, the presence of which is also indicated by the high values of the $[\mathrm{OI}] / \mathrm{H} \alpha$ ratio. High-velocity dispersions an enhanced $[\mathrm{OI}] / \mathrm{H} \alpha$ ratio in the stripped gas are also found in other galaxies that experience ram-pressure stripping, such as ESO 137-001 (Fumagalli et al. 2014) or the galaxies of the Shapley supercluster described in Merluzzi et al. (2016).

Based on our kinematical decomposition, we evaluated from the $\mathrm{H} \alpha$ luminosity of the stripped component that the interaction efficiently stripped $\approx 10^{9} M_{\odot}$ of ionized gas, consistent with the mass loss of $\approx 10^{9} M_{\odot}$ implied by the HI deficiency parameter $\left(D e f_{\mathrm{HI}}=0.23\right.$; Giovanelli \& Haynes 1985) measured by Gavazzi (1989). Therefore, despite the high mass and the edgeon configuration of UGC 6697, the stripping remains efficient.

The lower mass companion galaxy of UGC 6697, CGCG $97087 \mathrm{~N}$, is very close in projection to UGC 6697 $(\approx 14 \mathrm{kpc})$, and only the recent deep Subaru observations (Yagi et al. 2017) unveiled a double tail trailing behind the galaxy that is connected to the disk of UGC 6697. Owing to the sensitivity of MUSE, we clearly detect both tails that connect to the gas of UGC 6697, but in the velocity space, they remain 
well separated. The tails also indicate that CGCG $97087 \mathrm{~N}$ shows the effects of ram pressure from the ICM of the cluster while transiting in the cluster, similarly to UGC 6697. In addition, CGCG $97087 \mathrm{~N}$ was detected in X-rays (although no tails are observed, see Fig. 5) by Sun \& Vikhlinin (2005), who speculated that the X-ray emission arises from the gas heated by active star formation triggered by the tidal force of UGC 6697. The large difference between the recessional velocity of UGC 6697 and CGCG $97087 \mathrm{~N}\left(\Delta(c z) \approx 800 \mathrm{~km} \mathrm{~s}^{-1}\right)$ suggests that, if any, only a mild gravitational interaction occurred, consistent with the absence of stellar streams that connect the two galaxies along the gaseous tails even in the deepest data available of the Subaru. However, our non-parametrical analysis of the $\mathrm{H} \alpha$ flux reveals that the tails of CGCG $97087 \mathrm{~N}$ deeply penetrate the signal of the main body of UGC 6697. Moreover, in the [NII]/H $\alpha$ map, the tails of CGCG $97087 \mathrm{~N}$ have higher ratio values and connect perfectly to the only region of the stripped gas of UGC 6697 with such a $[\mathrm{NII}] / \mathrm{H} \alpha$ value. At the same time, the velocity dispersion of the tails appears connected to that of UGC 6697. These indicators are consistent with a past galaxy-galaxy interaction that shocked the gaseous component of both galaxies (enhanced $[\mathrm{NII}] / \mathrm{H} \alpha$ ), while leaving the stellar distributions unaltered. The impact between the galaxies occurred on almost perpendicular orbits, as indicated by the directions of the tails. Hence, the hydrodynamic interaction must have been short and the ram pressure on CGCG $97087 \mathrm{~N}$ only mildly enhanced for a very short time. We further speculate that this interaction possibly helped in keeping the stripping process of UGC 6697 efficient by perturbing its potential well.

Other gravitational interactions, for instance, with the giant elliptical NGC 3842, cannot be excluded a priori. The deep Subaru data (see Fig. 1) indeed highlight the proximity of one of the two giant E galaxies of Abell 1367, NGC 3842, whose recessional velocity differs from UGC 6697 by only $\approx 500 \mathrm{~km} \mathrm{~s}^{-1}$. This galaxy lies at a projected distance of $\approx 98 \mathrm{kpc}$ from the center of UGC 6697. The tail of UGC 6697 suggests a diagonal motion from the NW to the SE, while the bent jets of NGC 3842 (a narrow angle tail, NAT, galaxy) indicates a W-E motion. The small difference in the recessional velocities of the two galaxies and their small projected distance suggests a long interaction time. However, the real-space relative distances, relative velocities, and orbits are unknown, which prevents a reliable determination of parameters such as the time of encounter or the truncation radius, which would quantify the degree of the tidal perturbation. Given the mild asymmetries in the NW back of UGC 6697, we cannot completely exclude that a contribution from the tidal field of NGC 3842 is helping the stripping process. However, the absence of clear stellar streams, tidal tails, and shells in and around the galaxy disfavors the idea that gravitational interactions play a major role in this process.

\subsection{The tail(s)}

In our observations, the properties of the tails belonging to UGC 6697 and CGCG 97087N are different. In particular, the tail of UGC 6697 harbors many HII regions neatly distributed along preferential trails, while the tail of CGCG $97087 \mathrm{~N}$ does not. The kinematics of the two tails show a striking difference between the two galaxies: extended regions of the gas that retains small velocity dispersions $\left(<50 \mathrm{~km} \mathrm{~s}^{-1}\right)$ in the tail of UGC 6697. The typical velocity dispersions in the double tails of CGCG $97087 \mathrm{~N}$ are on the order $80-100 \mathrm{~km} \mathrm{~s}^{-1}$, while in UGC 6697 the gas associated with the trailing HII regions has typical velocity dispersions below $50 \mathrm{~km} \mathrm{~s}^{-1}$. At the same time,

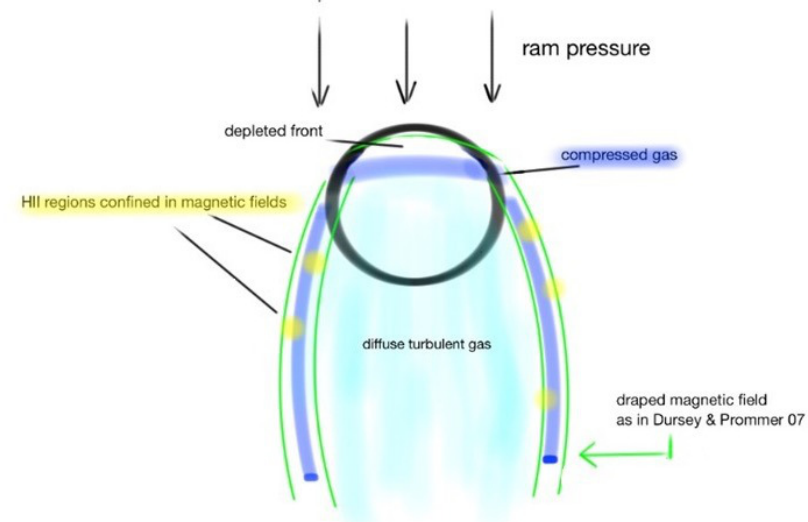

Fig. 17. Cartoon of the derived toy model for UGC 6697.

in the tails of CGCG $97087 \mathrm{~N}$, the $[\mathrm{OI}] / \mathrm{H} \alpha$ and $[\mathrm{NII}] / \mathrm{H} \alpha$ are highly enhanced with respect to the ionized gas in the whole frame, suggesting the presence of shocked gas and turbulent motions.

The gas stripped from UGC 6697 also shows regions where the $[\mathrm{OI}] / \mathrm{H} \alpha$ ratio suggests that shocks are heating the gas, but the $[\mathrm{NII}] / \mathrm{H} \alpha$ ratio of the stripped gas is on average low, with the exception of the region of possible interaction with CGCG $97097 \mathrm{~N}$. Taking into account the models at different metallicities of Kewley et al. (2001), we interpret the low values of $[\mathrm{NII}] / \mathrm{H} \alpha$ as a lower metallicity of the stripped diffuse gas. This is consistent with a picture where the stripping acted efficiently on the most external gas of the galaxy, which is characterized by lower metallicities $\left(\approx 0.4 Z_{\odot}\right)$, consistent with the metallicity gradients observed in spiral Sb-Sc galaxies (González Delgado et al. 2015).

Our results are in line with the result observed in the ESO 137-001 (Fumagalli et al. 2014; Fossati et al. 2016) galaxy, where the stripped tail shows enhanced $[\mathrm{OI}] / \mathrm{H} \alpha$ and $[\mathrm{NII}] / \mathrm{H} \alpha$ as well as high-velocity dispersions, with the exception of zones harboring HII regions. Consistent results have recently also been found in two galaxies showing tails of ram-pressure stripping within the GASP survey (Poggianti et al. 2017; Bellhouse et al. 2017). Hence, the star formation in the tail appears to be associated with the less turbulent component of the stripped gas. The properties (i.e., line ratios and velocity dispersions) of the HII regions that formed outside the galaxy are not different from the HII regions in the galaxy. Apparently, these are formed only along two preferential trails, which suggests that in these regions cooling and collapse might be favored by a higher gas density and magnetic confinement. In Fig. 17 (left panel) we plot a cartoon of our toy model of the UGC 6697 system that we developed in the light of recent magnetohydrodynamical simulations by Ruszkowski et al. (2014).

In this model, UGC 6697 is traveling at high speed near the core of Abell 1367 in a nearly edge-on configuration. This configuration should disfavor ram-pressure stripping, but in contrast, the stripping may be enhanced and favored by the interaction with CGCG $97087 \mathrm{~N}$ and by the interaction of the disk surface with the magnetic fields sliding past the ISM/ICM interface (Ruszkowski et al. 2014). This configuration of impact is similar to the configurations explored in the simulations by Ruszkowski et al. (2014) of a $\sim 10^{11} M_{\odot}$ stellar mass galaxy that experienced magneto-hydrodynamic interaction with an ICM whose physical parameters match those of observed clusters $\left(n_{\mathrm{e}} \sim 0.5 \times 10^{-3} \mathrm{~cm}^{-3} ; T \sim 7 \mathrm{keV} ; B \sim 1.7 \mu \mathrm{G}\right)$ at a distance of $\approx 0.6 \mathrm{Mpc}$ from the center of the cluster. These simulations 
showed that magnetic fields induce a filamentary shape of the stripped material that would otherwise appear as clumpy. In particular, two magnetized tails with enhanced gas density arise in the simulations, favoring cooling and collapse with respect to the turbulent and less dense gas in the other filaments. We speculate that in UGC 6697 we view a very similar phenomenon where the motion of the galaxy through the magnetized ICM drapes the magnetic fields lines (Dursi \& Pfrommer 2008) and produces two magnetized trails similar to the tails in the simulations of Ruszkowski et al. (2014). At the front of the galaxy, ram pressure compresses the gas to the NW and leaves a depleted front. At the same time, along the body of the galaxy, at the interface between the ISM and the ICM, Kelvin-Helmotz instabilities and magnetic scraping rip the gas from the disk. Subsequently, the ram-pressure wind pushes this gas to the back of the galaxy. In the magnetized tails, the gas is compressed, cools, and forms stars.

Acknowledgements. The authors would like to thank the referee for the constructive criticism. We also thank M. Sun, who kindly shared his X-ray data with us. This research has made use of the GOLDmine database (Gavazzi et al. 2003 2014b) and of the NASA/IPAC Extragalactic Database (NED), which is operated by the Jet Propulsion Laboratory, California Institute of Technology, under contract with the National Aeronautics and Space Administration. M. Fossati acknowledges the support of the Deutsche Forschungsgemeinschaft via Projects WI 3871/1-1, and WI 3871/1-2. M. Fumagalli acknowledges support by the Science and Technology Facilities Council [grant number ST/P000541/1]. For access to the codes and advanced data products used in this work, please contact the authors or visit https://github.com/mifumagalli/mypython/tree/ master/ifu. Raw data are publicly available via the ESO Science Archive Facility.

\section{References}

Allen, M. G., Groves, B. A., Dopita, M. A., Sutherland, R. S., \& Kewley, L. J. 2008, ApJS, 178, 20

Baldwin, J. A., Phillips, M. M., \& Terlevich, R. 1981, PASP, 93, 5

Bekki, K., \& Couch, W. J. 2003, ApJ, 596, L13

Binggeli, B., Tammann, G. A., \& Sandage, A. 1987, AJ, 94, 251

Binney, J., \& Tremaine, S. 1987 (Princeton: Princeton University Press), 747

Bellhouse, C., Jaffe, Y. L., Hau, G. K. T., et al. 2017, ApJ, 844, 49

Bliton, M., Rizza, E., Burns, J. O., Owen, F. N., \& Ledlow, M. J. 1998, MNRAS, 301, 609

Boselli, A., \& Gavazzi, G. 2006, PASP, 118, 517

Boselli, A., \& Gavazzi, G. 2014, A\&ARv, 22, 74

Boselli, A., Gavazzi, G., Combes, F., Lequeux, J., \& Casoli, F. 1994, A\&A, 285 , 69

Boselli, A., Boissier, S., Cortese, L., \& Gavazzi, G. 2008, ApJ, 674, 742

Boselli, A., Cuillandre, J. C., Fossati, M., et al. 2016, A\&A, 587, A68

Cappellari, M., \& Emsellem, E. 2004, PASP, 116, 138

Cardelli, J. A., Clayton, G. C., \& Mathis, J. S. 1989, ApJ, 345, 245

Chabrier, G. 2003, PASP, 115, 763

Chung, A., van Gorkom, J. H., Kenney, J. D. P., Crowl, H., \& Vollmer, B. 2009, AJ, 138, 1741

Consolandi, G., Gavazzi, G., Fumagalli, M., Dotti, M., \& Fossati, M. 2016, A\&A, 591, A38

Courteau, S., Dutton, A. A., van den Bosch, F. C., et al. 2007, ApJ, 671, 203

Cowie, L. L., \& McKee, C. F. 1977, ApJ, 211, 135

Curti, M., Cresci, G., Mannucci, F., et al. 2017, MNRAS, 465, 1384

Draine, B. T. 2011, Physics of the Interstellar and Intergalactic Medium (Princeton University Press)

Dressler, A. 1980, ApJ, 236, 351

Duc, P.-A., Cuillandre, J.-C., Karabal, E., et al. 2015, MNRAS, 446, 120

Dursi, L. J., \& Pfrommer, C. 2008, ApJ, 677, 993

Ebeling, H., Stephenson, L. N., \& Edge, A. C. 2014, ApJ, 781, L40

Fontanot, F., De Lucia, G., Monaco, P., Somerville, R. S., \& Santini, P. 2009, MNRAS, 397, 1776

Fossati, M., Gavazzi, G., Boselli, A., \& Fumagalli, M. 2012, A\&A, 544, A128
Fossati, M., Fumagalli, M., Boselli, A., et al. 2016, MNRAS, 455, 2028

Fritz, J., Moretti, A., Poggianti, B., et al. 2017, ApJ, submitted [arXiv: 1704.05088]

Fruscione, A., \& Gavazzi, G. 1990, BAAS, 22, 746

Fumagalli, M., Gavazzi, G., Scaramella, R., \& Franzetti, P. 2011, A\&A, 528, A46

Fumagalli, M., Fossati, M., Hau, G. K. T., et al. 2014, MNRAS, 445, 4335

Fumagalli, M., Haardt, F., Theuns, T., et al. 2017, MNRAS, 467, 4802

Gavazzi, G. 1978, A\&A, 69, 355

Gavazzi, G. 1987, ApJ, 320, 96

Gavazzi, G. 1989, ApJ, 346, 59

Gavazzi, G. 2009, Rev. Mex. Astron. Astrofis. Conf. Ser., 37, 72

Gavazzi, G., \& Jaffe, W. 1987, A\&A, 186, L1

Gavazzi, G., Tarenghi, M., Jaffe, W., Boksenberg, A., \& Butcher, H. 1984, A\&A, 137,235

Gavazzi, G., Contursi, A., Carrasco, L., et al. 1995, A\&A, 304, 325

Gavazzi, G., Marcelin, M., Boselli, A., et al. 2001, A\&A, 377, 745

Gavazzi, G., Boselli, A., Donati, A., Franzetti, P., \& Scodeggio, M. 2003, A\&A 400,451

Gavazzi, G., Savorgnan, G., Fossati, M., et al. 2013, A\&A, 553, A90

Gavazzi, G., Franzetti, P., \& Boselli, A. 2014, ArXiv e-prints [arXiv: 1401.8123]

Giovanelli, R., \& Haynes, M. P. 1985, ApJ, 292, 404

González Delgado, R. M., García-Benito, R., Pérez, E., et al. 2015, A\&A, 581, A103

Gunn, J. E., \& Gott, J. R., III 1972, ApJ, 176, 1

Jáchym, P., Combes, F., Cortese, L., Sun, M., \& Kenney, J. D. P. 2014, ApJ, 792, 11

Kauffmann, G., Heckman, T. M., Tremonti, C., et al. 2003, MNRAS, 346, 1055

Kenney, J. D. P., Geha, M., Jáchym, P., et al. 2014, ApJ, 780, 119

Kewley, L. J., Dopita, M. A., Sutherland, R. S., Heisler, C. A., \& Trevena, J. 2001, ApJ, 556, 121

Kregel, M., van der Kruit, P. C., \& Freeman, K. C. 2005, MNRAS, 358, 503

Larson, R. B., Tinsley, B. M., \& Caldwell, C. N. 1980, ApJ, 237, 692

Merluzzi, P., Busarello, G., Dopita, M. A., et al. 2016, MNRAS, 460, 3345

Moore, B., Katz, N., Lake, G., Dressler, A., \& Oemler, A. 1996, Nature, 379, 613

Nilson, P. 1973, Acta Universitatis Upsaliensis. Nova Acta Regiae Societatis Scientiarum Upsaliensis - Uppsala Astronomiska Observatoriums Annaler (Uppsala: Astronomiska Observatorium)

Noll, S., Kausch, W., Kimeswenger, S., et al. 2014, A\&A, 567, A25

Nulsen, P. E. J. 1982, MNRAS, 198, 1007

Osterbrock, D. E. 1989, Research supported by the University of California, John Simon Guggenheim Memorial Foundation, University of Minnesota (Mill Valley, CA: University Science Books), 422

Osterbrock, D. E., \& Ferland, G. J. 2006, Astrophysics of gaseous nebulae and active galactic nuclei, 2nd. edn. (Sausalito: University Science Books)

Piffaretti, R., Arnaud, M., Pratt, G. W., Pointecouteau, E., \& Melin, J.-B. 2011, A\&A, 534, A109

Poggianti, B. M., Fasano, G., Omizzolo, A., et al. 2016, AJ, 151, 78

Poggianti, B. M., Moretti, A., Gullieuszik, M., et al. 2017, ApJ, 844, 48

Read, J. I., Wilkinson, M. I., Evans, N. W., Gilmore, G., \& Kleyna, J. T. 2006, MNRAS, 366, 429

Rich, J. A., Kewley, L. J., \& Dopita, M. A. 2011, ApJ, 734, 87

Ruszkowski, M., Brüggen, M., Lee, D., \& Shin, M.-S. 2014, ApJ, 784, 75

Salpeter, E. E. 1955, ApJ, 121, 161

Sarzi, M., Falcón-Barroso, J., Davies, R. L., et al. 2006, MNRAS, 366, 1151

Scott, T. C., Usero, A., Brinks, E., et al. 2013, MNRAS, 429, 221

Siudek, M., Małek, K., Scodeggio, M., et al. 2017, A\&A, 597, A107

Storey, P. J., \& Zeippen, C. J. 2000, MNRAS, 312, 813

Sullivan, W. T., III, Bates, B., Bothun, G. D., \& Schommer, R. A. 1981, AJ, 86, 919

Sun, M., \& Vikhlinin, A. 2005, ApJ, 621, 718

Sun, M., Donahue, M., \& Voit, G. M. 2007, ApJ, 671, 190

Yagi, M., Komiyama, Y., Yoshida, M., et al. 2007, ApJ, 660, 1209

Yagi, M., Yoshida, M., Komiyama, Y., et al. 2010, AJ, 140, 1814

Yagi, M.,Yoshida, M., Gavazzi, G., et al. 2017, ApJ, submitted

Yoshida, M., Ohyama, Y., Iye, M., et al. 2004, AJ, 127, 90

Vazdekis, A., Sánchez-Blázquez, P., Falcón-Barroso, J., et al. 2010, MNRAS, 404, 1639

Wisnioski, E., Förster Schreiber, N. M., Wuyts, S., et al. 2015, ApJ, 799, 209

Zibetti, S., Charlot, S., \& Rix, H.-W. 2009, MNRAS, 400, 1181

Zwicky, F., Herzog, E., Wild, P., Karpowicz, M., \& Kowal, C. T. 1961-1968 (Pasadena: California Institute of Technology (CIT)) 